Portland State University

PDXScholar

1978

\title{
Washington County Mental Health Program Year End Report for Fiscal Year 1976-1977
}

Jennifer Dee Pockel

Portland State University

Follow this and additional works at: https://pdxscholar.library.pdx.edu/open_access_etds

Part of the Social Work Commons

Let us know how access to this document benefits you.

\section{Recommended Citation}

Pockel, Jennifer Dee, "Washington County Mental Health Program Year End Report for Fiscal Year 1976-1977" (1978). Dissertations and Theses. Paper 2667.

https://doi.org/10.15760/etd.2663

This Thesis is brought to you for free and open access. It has been accepted for inclusion in Dissertations and Theses by an authorized administrator of PDXScholar. Please contact us if we can make this document more accessible: pdxscholar@pdx.edu. 


\section{ABSTRACT}

Program evaluation is in its infancy at the Washington County Mental Health Program; it is only within the last two years that there has been any emphasis placed on evaluation activities. The first chapter in this practicum will identify and provide a context for the recent introduction of the management by objectives approach to process evaluation within the Washington County Mental Health Program as well as present a rationale for such an approach; the second chapter in this practicum is a presentation of results obtained from the first follow-up study to employ management objectives as an evaluation tool; the third chapter explores problems inherent in the nature and design of this type of evaluation procedure as well as program implications concerning the utility of results obtained from the study and recommendations for change in both approach and procedure. 


\title{
WASHINGTON COUNTY MENTAL HEALTH PROGRAM YEAR END \\ REPORT FOR FISCAL YEAR 1976-1977
}

\begin{abstract}
by
JENNIFER DEE POCKEL

A practicum submitted in partial fulfillment of the requirement for the degree of
\end{abstract}

MASTER OF SOCIAL WORK

Portland State University

1978 
TO THE OFFICE OF GRADUATE STUDIES AND RESEARCH:

The Advisor approves the practicum of Jennifer Dee Pockel presented May 10, 1978

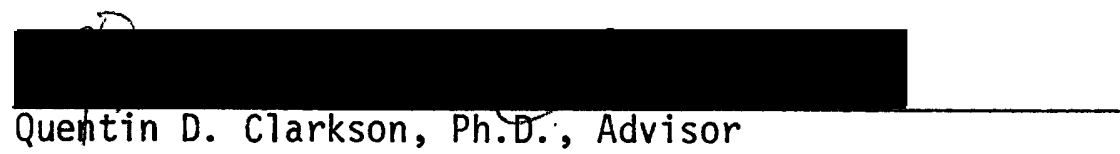


TABLE OF CONTENTS

CHAPTER

PAGE

I INTRODUCTION . . . . . . . . . . . . . . . 1

II WASHINGTON COUNTY MENTAL HEALTH PROGRAM YEAR
END REPORT, FISCAL YEAR $1976-1977 \ldots \ldots 6$

Comprehensive Program Narrative ... . . . 6

Alcohol Program

Drug Program

Developmental Disabilities Program

Mental and Emotional Disturbances Program

Condensed Program Statistics . . . . . . 11

Administrative Statistics ......... 16

Alcohol Program Statistics; Subcontract Agency

Reports .............. 20

Drug Program Statistics; Subcontract Agency Reports ............ 47

Developmental Disabilities Program Statistics; Subcontract Agency Reports....... . 47

Mental \& Emotional Disturbances Program

Statistics; Subcontract Agency Reports . . . . 49

III CONCLUSIONS AND RECOMMENDATIONS . . . . . . . 62

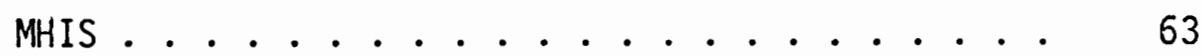

Alternatives to MHIS ......... . . 65

Decision-making Process ........ 65

Recommendations for Change ........ 67

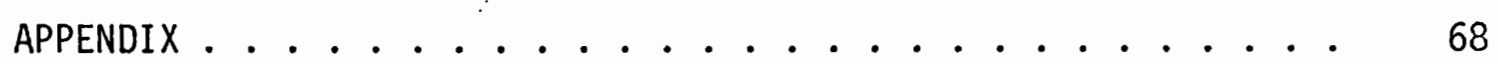




\section{CHAPTER I}

\section{INTRODUCTION}

At the present time, evaluation activities, occurring within the Washington County Mental Health Program, are performed by administrative core staff members, private consultants, and directors of individual service providers (subcontract agencies). The evaluation efforts relate strictly to process objectives; outcome evaluation is virtually nonexistent. Needs assessments are few; for the most part evaluation focuses on services provided to clients who have already been identified and currently participate in the formal mental health system. Needs assessments and utilization studies, encompassing the bulk of current program evaluation efforts, are most frequently undertaken by agents outside of the service-providing agency.

Fiscal year 1976-1977 marked the beginning of a major revision in evaluation and program accountability for the Washington County Mental Health Program. Each year administrative staff members construct a Comprehensive Mental Health Plan; this plan is required by ORS 430.630 and constitutes the basis for negotiation with the Oregon State Mental Health Division for the funding of mental health services in Washington County. Traditionally, this plan has included data from needs assessments, descriptions of existing service elements, and priorities for service development in each of the program areas: Administration, Alcohol (A), Drug (D), Developmental Disabilities (DD), and Mental and 
Emotional Disturbances (MED). Each program area, within the plan, contains: (1) at risk indicators and target populations in need of services, (2) program descriptions of existing mental health resources available to Washington County residents, (3) prioritized program development plans for meeting identified service delivery needs, and (4) a description of agencies and programs currently providing services as part of the Washington County Mental Health Program (subcontract agencies).

The Comprehensive Mental Health Plan for fiscal year 1976-1977, in addition to these traditional content areas, includes a series of management by objectives (MBO) budget packages. These management by objectives budget packages outline, by subcontract agency, by service element, by target population, how many families and individuals will be served in each service delivery area and at what cost to the program. The content of these management objectives represents a consolidation of information contributed by the mental health "specialists" (members of the administrative core staff) in each program area, members of community advisory groups, and directors and agency staff within each of the subcontract agencies (service providers).

Management objectives were submitted to the administrative core staff team by the heads of the various subcontract agencies. The administrative "specialists" in each program area then worked, with subcontract agency directors, to further define, modify, and interface these objectives. When the objectives were satisfactory to both parties, they were implemented as part of the MBO budget package peculiar to that subcontract agency. During this phase of developing management 
objectives, a growing awareness and appreciation concerning the difficulty associated with the writing of clear, concise, easily measured service objectives served to promote and direct treatment planning energy and thought on the part of both subcontract agency personnel and Washington County administrators.

Many of the management objectives, developed for fiscal year 1976-1977, are weak (e.g., we will inform 30 percent of Washington County of local alcohol resources) in terms of specificity and measurability; others are well detailed (e.g., we will provide brief inpatient services for twenty county residents with MED problems). In general, objectives from the DD program area tend to be better written and easier to measure than those from administration, A, D, and MED program areas. There are many reasons for this, including the treatment modality most often employed in the DD program area (behavior modification), which most easily lends itself to quantifiable treatment evaluation approaches. In many instances, the DD program area format serves as a model for the other program areas. The administrative objectives tend to be the weakest, least measurable; this may be due, in part, to the fact that data, relevant to these objectives and required for follow-up, are not collected by the state management information system (MHIS) or in any other systematized manner.

The rationale behind and intent of the MBO approach is to hold each agency, involved in the Washington County Mental Health Program, responsible for negotiating and following through with a contract for service delivery that is easily defined, measurable, and mutually satisfactory to both agency director and Washington County Mental Health 
Program area specialist. The MBO budget packages represent a purchase of treatment agreement similar to sales contracts prevalent in the business community. They are designed to promote fiscal accountability, greater visibility of treatment options, a clarity of purpose and direction within the mental health system, as well as support a more reliable method of tracking clients through service delivery elements.

Each agency, along with adopting an MBO budget package agreement, is required to collect and submit data (on a twice yearly basis), relating to the agreed-upon objectives, to the Washington County Mental Health Program Specialist in the appropriate program area. Data are submitted both through the State Mental Health Information System and through the agencies' own record keeping systems. Washington County Mental Health administrative staff members process the evaluation and feed it back to the agency directors with comment. In this manner, agency directors are held accountable for contracts which are not adequately upheld and may experience a modification in the amount of county funds available to them.

In the "Washington County Year End Report for Fiscal Year 19761977" (Chapter II of this practicum), data from both agency record keeping systems and MHIS, relating to the management by objectives budget packages outlined in the "Comprehensive Mental Health Plan," have been extracted, consolidated, and condensed in order to examine and contrast the projected and actual service utilization rates. This report was compiled, by this author, for use, by the Washington County Mental Health Program administrators, in renegotiating and refining subcontract agency MBO packages, in obtaining funding from the Washington County 
Commissioners and in obtaining additional funding from the Oregon State Mental Health Division. In addition, it has been useful in serving to focus treatment efforts, defining treatment objectives, triggering cost/ benefit discussions, and tracking mental health client populations within the mental health system.

Chapter II of this practicum is the "Washington County Year End Report for Fiscal Year 1976-1977." Some problems are inherent in the nature and design of this type of evaluation as well as the available record keeping systems; they are discussed in Chapter III along with program implications arising from the utilization of this type of evaluation effort and recommendations for improvement in future evaluation activities of this kind. 


\title{
CHAPTER II
}

\author{
WASHINGTON COUNTY MENTAL HEALTH PROGRAM \\ YEAR END REPORT, FISCAL YEAR 1976-1977 \\ COMPREHENSIVE PROGRAM NARRATIVE
}

The Washington County Mental Health Program served a total of 7,429 Washington County residents during the fiscal year 1976-1977. Three thousand one hundred thirty-eight were served through direct services, and 4,291 were served through indirect services. This is not an unduplicated count. The services were provided in four program areas: Alcohol, Drug, Developmental Disabilities, and Mental and Emotional Disturbances. County adminịtrative staff coordinated the service delivery across program areas, was responsible for program administration, program development, preparation and monitoring of contracts, as well as program evaluation activities.

Eighteen service elements, or categories of service delivery, were provided in fiscal year 1976-1977. Direct service elements, involving face-to-face contact, are as follows, along with the number of clients served in each during the year.

2,081 Outpatient

19 Inpatient

84 24-hour Emergency "Beeper" Consultation

56 Substance Abuse-related Medical Treatment

38 TMR Preschool 
119 TMR Classroom

64 MR/DD Adult Activity Center

10 Residential Treatment

52 Socialization and Day Treatment

14 Homemaker

587 Commitment and Diversion

14 Aftercare

Indirect service delivered, including consultation not requiring opening of a case, were as follows.

1,398 Information and Assistance

758 Outreach and Advocacy

1,965 Consultation and Education

170 Continuity and Coordination

Washington County contracted with eighteen local agencies or "subcontract agencies," to provide the above mental health services.

Below is a quantitative breakdown by program area. Again, the complication of duplications in the count arises; there is always the possibility that one person was served in more than one service element.

\section{Alcohol Program}

The Alcohol Program included seven subcontract agencies as well as the Washington County :Alcohol Specialist. These agencies were: Substance Abuse Council, Tri-County Community Council, Washington County Youth Contact, Washington County Community Corrections, Native American Rehabilitation Association, Tualatin Valley Mental Health Center, and Centro Cultural. Two thousand forty-three individuals and families were served in eleven service elements, as follows. 
329 Outpatient

21 24-hour Emergency "Beeper" Consultation

37 Substance Abuse-related Medical Treatment

469 Information and Assistance

330 Outreach and Advocacy

857 Consultation and Education

Three hundred eight-seven clients received direct services, and 1,656 clients received indirect services.

\section{Drug Program}

The Drug Program involved no subcontract agencies. The program incorporated CODA loan staff, HB 2145 resources, drug diversion resources, and the Washington County Drug Specialist and related staff. One thousand four hundred eighty individuals and families were served in seven service elements.

170 Outpatient

11 24-hour Emergency "Beeper" Consultation

19 Substance Abuse-related Medical Treatment

641 Information and Assistance

428 Outreach and Advocacy

63 Consultation and Education

148 Continuity and Coordination

Two hundred $\mathrm{clients}$ received direct services, and 1,280 $\mathrm{clients}$ received indirect services. 
Developmental Disabilities Program

The Developmental Disabilities Program included seven subcontract agencies, as well as the Washington County Developmental Disabilities staff, and the County Child Development Program. These agencies were: Beaverton Preschool, Beaverton TMR, Forest Grove TMR, Good Shepherd TMR, Edwards Activity Center, Edwards Group Home, and TVW, Inc. One thousand twenty-five individuals and families were served in seven service elements.

13 24-hour Emergency "Beeper" Consultation

38 TMR Preschool

119 TMR Classroom

64 MR/DD Adult Activity Center

10 Residential Treatment

Information and Assistance (count not available)

781 Consultation and Education

Two hundred forty-eight clients received direct services, and $781 \mathrm{cli-}$ ents received indirect services.

Mental and Emotional Disturbances

Program

The Mental and Emotional Disturbances Program included four subcontract agencies, as well as the Washington County MED Specialist, the Involuntary Commitment Program, a Brief Inpatient Program at Cedar Hills Hospital, and an Alternatives to Commitment Program. These MED agencies were: Lutheran Family Services, Metropolitan Family Services, Tualatin Valley Mental Health Center, and TVW, Inc. Day Treatment. Two thousand 
five hundred ninety-three individuals and families were served in ten service elements.

1,582 Outpatient

19 Inpatient

39 24-hour Emergency "Beeper" Consultation

52 Day Treatment

14 Homemaker

587 Commitment and Diversion

14 Aftercare

288 Information and Assistance

264 Consultation and Education

22 Continuity and Coordination

Two thousand two hundred ninety-seven clients received direct services, and more than 286 clients received indirect services. 


\section{WASHINGTON COUNTY MENTAL HEALTH PROGRAM: END OF YEAR EVALUATION}

PROGRAM AREA: Across All Program Areas

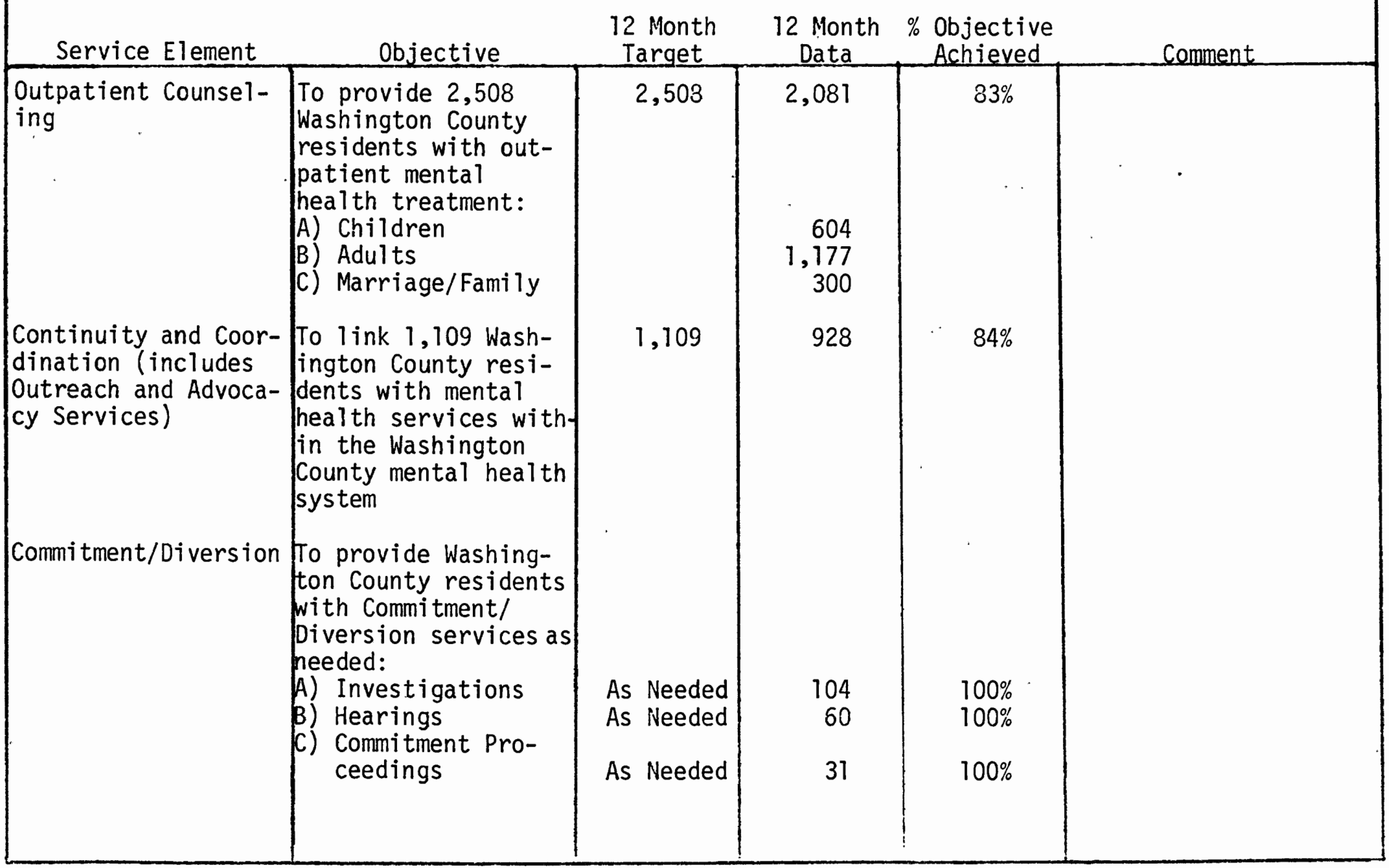


WASHINGTON COUNTY MENTAL HEALTH PROGRAM: END OF YEAR EVALUATION

PROGRAM AREA: Across A11 Program Areas

\begin{tabular}{|c|c|c|c|c|c|}
\hline Service Element & Objective & $\begin{array}{l}12 \text { Month } \\
\text { Target }\end{array}$ & $\begin{array}{l}12 \text { Month } \\
\text { Data }\end{array}$ & $\begin{array}{c}\% \text { Objective } \\
\text { Achieved }\end{array}$ & Comment \\
\hline $\begin{array}{l}\text { TMR CTassroom Ser- } \\
\text { vices and Adult MR/ } \\
\text { DD Activity Centers }\end{array}$ & $\begin{array}{l}\text { To provide } 242 \text { Wash- } \\
\text { ington County resi- } \\
\text { dents with TMR and } \\
\text { Activity Center } \\
\text { treatment: } \\
\text { A) TMR--Preschool } \\
\text { B) TMR--School Age } \\
\text { C) Activity--Adult }\end{array}$ & $\begin{array}{r}36 \\
134 \\
72\end{array}$ & $\begin{array}{r}38 \\
119 \\
64\end{array}$ & $\begin{array}{r}105 \% \\
89 \% \\
89 \%\end{array}$ & \\
\hline $\begin{array}{l}\text { MR Residential Treat. } \\
\text { ment }\end{array}$ & $\begin{array}{l}\text { To provide } 8 \text { wash- } \\
\text { ington County resi- } \\
\text { dents with residen- } \\
\text { tial treatment }\end{array}$ & 8 & 10 & $125 \%$ & \\
\hline Day Treatment & $\begin{array}{l}\text { To provide } 60 \text { Wash- } \\
\text { ington County resi- } \\
\text { dents with Adult/Day } \\
\text { Treatment services }\end{array}$ & 60 & 52 & $87 \%$ & \\
\hline Medical Services & $\begin{array}{l}\text { To provide Washing- } \\
\text { ton County residents } \\
\text { with: } \\
\text { A) } 645 \text { Antabuse Doses } \\
\text { B) } 36 \text { Physicals } \\
\text { C) Detoxification } \\
\text { (10) }\end{array}$ & $\begin{array}{r}645 \\
36 \\
10\end{array}$ & $\begin{array}{r}340 \\
37 \\
19\end{array}$ & $\begin{array}{r}52 \% \\
103 \% \\
190 \%\end{array}$ & \\
\hline
\end{tabular}


WASHINGTON COUNTY MENTAL HEALTH PROGRAM: END OF YEAR EVALUATION

PROGRAM AREA: Across All Program Areas

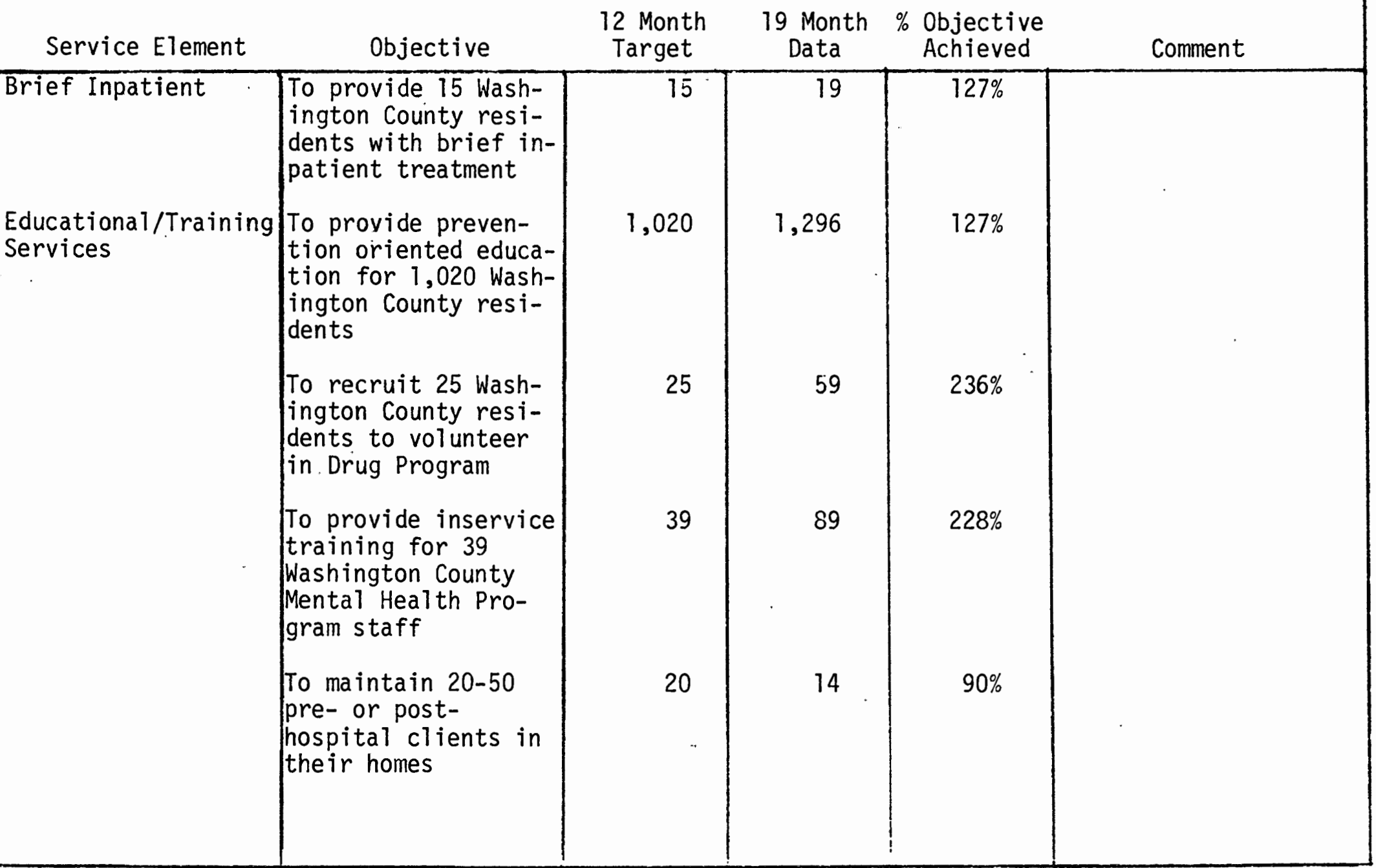




\begin{tabular}{|c|c|c|c|c|c|}
\hline \multicolumn{6}{|c|}{ PROGRAM AREA: Across A11 Program Areas } \\
\hline Consultation & \begin{tabular}{|l|} 
To provide 1,315 \\
hours of consulta- \\
tion to Washington \\
County Mental Health \\
agencies \\
To contact 100 agen- \\
cies outside of \\
Washington County \\
regarding their Sub- \\
stance Abuse Program
\end{tabular} & 100 & $1,142^{\star}$ & $120 \%$ & . \\
\hline $\begin{array}{l}\text { Information and Re- } \\
\text { ferral }\end{array}$ & $\begin{array}{l}\text { To provide informa- } \\
\text { tion and referral } \\
\text { services for } 1,243 \\
\text { Washington County } \\
\text { residents }\end{array}$ & 1,243 & 1,398 & $112 \%$ & \\
\hline 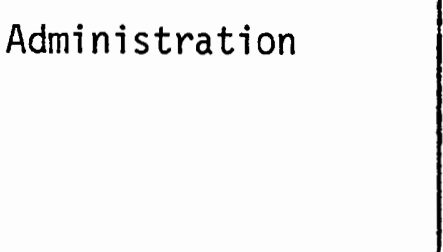 & $\begin{array}{l}\text { Develop and imple- } \\
\text { ment coordinated de- } \\
1 \text { ivery system which } \\
\text { unifies MED, A\&D, } \\
\text { and MR/DD services }\end{array}$ & iv/ & 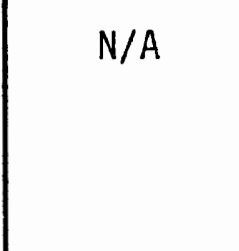 & N/A & \\
\hline $\begin{array}{l}\text { *This objective } \\
\text { "hour" units to "pers } \\
\text { "hours" of consultat } \\
\text { served in the summary }\end{array}$ & $\begin{array}{l}\text { represents hours, no } \\
\text { son" units reflects an } \\
\text { on. For this reason } \\
\text { and the totals in th }\end{array}$ & $\begin{array}{l}t \text { persons or } \\
\text { estimate or } \\
\text { there is a } \\
\text { e 12-month }\end{array}$ & $\begin{array}{l}\text { agencies s } \\
\text { ly. One "p } \\
\text { discrepancy } \\
\text { ata column }\end{array}$ & $\begin{array}{l}\text { erved. There } \\
\text { erson" repres } \\
\text { between the } \\
\text { on this page. }\end{array}$ & $\begin{array}{l}\text { fore, conversion from } \\
\text { ents approximately two } \\
\text { total number of clients }\end{array}$ \\
\hline
\end{tabular}




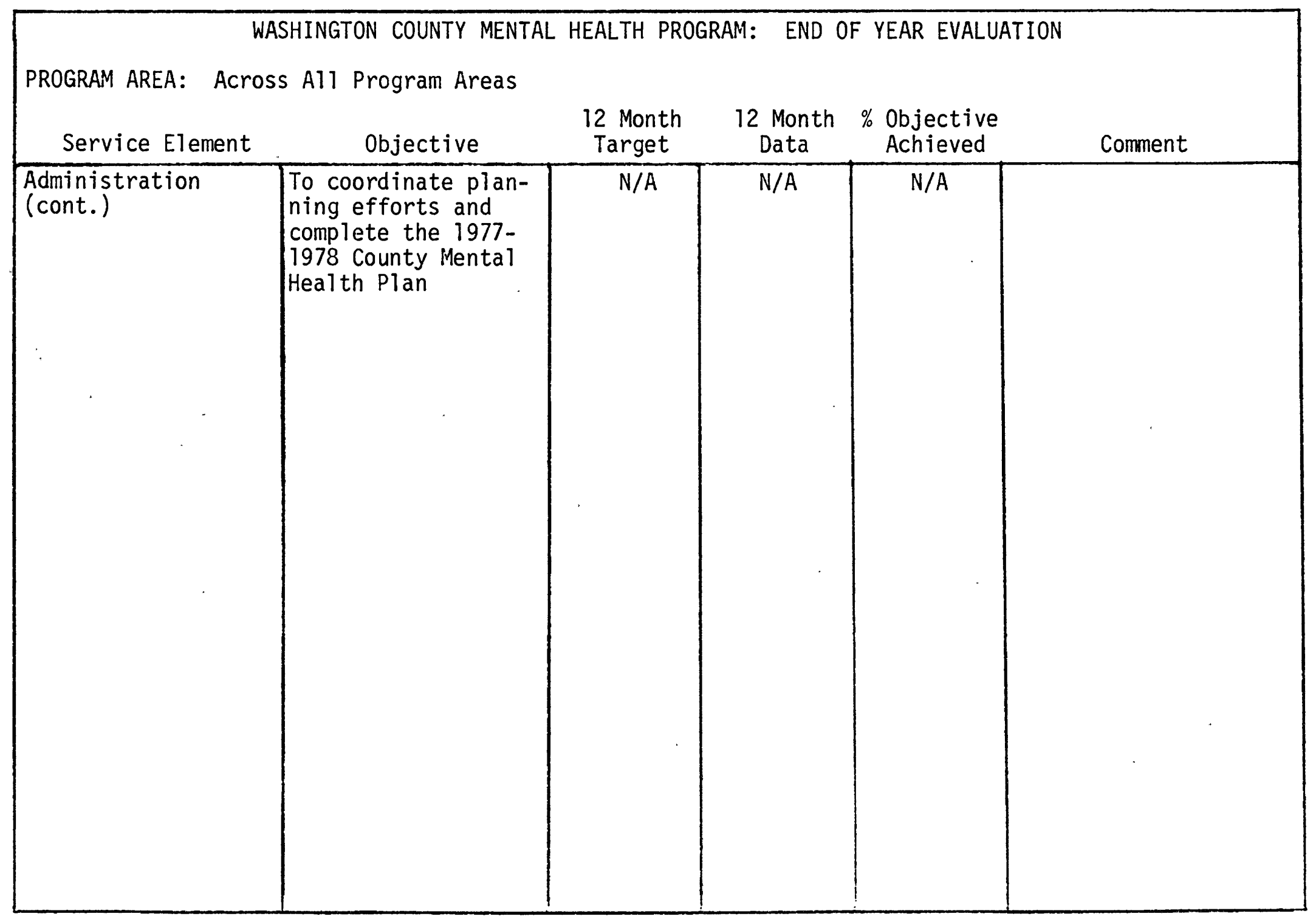

$\vec{G}$ 


\begin{tabular}{|c|c|c|c|c|c|}
\hline \multicolumn{6}{|c|}{$\begin{array}{l}\text { WASHINGTON COUNTY MENTAL HEALTH PROGRAM: END OF YEAR EVALUATION } \\
\text { PROGRAM AREA: Administration }\end{array}$} \\
\hline Service Element & Objective & $\begin{array}{l}12 \text { Month } \\
\text { Target }\end{array}$ & $\begin{array}{l}12 \text { Month } \\
\text { Data }\end{array}$ & $\begin{array}{l}\% \text { Objective } \\
\text { Achieved }\end{array}$ & Comment \\
\hline $\begin{array}{l}\text { Administrative Ser- } \\
\text { vices }\end{array}$ & $\begin{array}{l}\text { Develop and imple- } \\
\text { ment coordinated de- } \\
\text { livery system which } \\
\text { unifies MED, A\&D, } \\
\text { and MR/DD services } \\
\text { Prepare and monitor } \\
\text { subcontract agency } \\
\text { agreements for MED, } \\
\text { MR/DD, and A\&D ser- } \\
\text { vices } \\
\\
\text { Assist county advi- } \\
\text { sory boards in per- } \\
\text { forming their func- } \\
\text { tions }\end{array}$ & & . & & $\begin{array}{l}\text { After care CETA pro- } \\
\text { gram implemented. Con } \\
\text { sultation with CSD and } \\
\text { AFS and intra-agency } \\
\text { screenings for day } \\
\text { treatment and group } \\
\text { home established. } \\
\text { Intra-MED agency coor- } \\
\text { dination facil itated. } \\
\text { } 19 \text { subcontract agree- } \\
\text { ments for service ele- } \\
\text { ments developed with } \\
13 \text { separate agencies. } \\
\text { Monthly fiscal moni- } \\
\text { toring, UR's quarterly } \\
\text { for most agencies, } \\
\text { client involvement } \\
\text { follow-up and six } \\
\text { month and annual MBO } \\
\text { evaluation } \\
\text { M.H. Director, A\&D Spe- } \\
\text { cialists, MED and MR/ } \\
\text { DD Specialists staffed } \\
\text { the M.H. Advisory } \\
\text { Board, Substance Abuse } \\
\text { Council, Mental and }\end{array}$ \\
\hline
\end{tabular}




\begin{tabular}{|c|c|c|c|c|c|}
\hline \multicolumn{6}{|c|}{ PROGRAM AREA: Administration } \\
\hline $\begin{array}{l}\text { Administrative Ser- } \\
\text { vices } \\
\text { (cont.) }\end{array}$ & $\begin{array}{l}\text { Assist county advi- } \\
\text { sory boards in per- } \\
\text { forming their func- } \\
\text { tions } \\
\text { (cont.) } \\
\text { To coordinate plan- } \\
\text { ning efforts and } \\
\text { complete the 1977-78 } \\
\text { County Mental Health } \\
\text { Plan } \\
\\
\text { To Assist Board of } \\
\text { Commissioners in mak } \\
\text { ing policy decisions } \\
\text { regarding mental } \\
\text { health programs }\end{array}$ & & & . & $\begin{array}{l}\text { Emotional Health Com- } \\
\text { mittee on a regular } \\
\text { basis } \\
\text { The 1977-78 County } \\
\text { M.H. Plan and involve- } \\
\text { ment of advisory boards } \\
\text { and others in mental } \\
\text { health planning was } \\
\text { outstanding. Major } \\
\text { planning was completed } \\
\text { on emergency service, } \\
\text { M.H. geriatric ser- } \\
\text { vices, services to } \\
\text { children, services with } \\
\text { CSD, mental health } \\
\text { needs in schools, and } \\
\text { inpatient services. } \\
\text { The plan was completed } \\
\text { and submitted to the } \\
\text { MHD in June } 1977 . \\
\text { Board of Commissioners } \\
\text { continued involvement } \\
\text { in mental health and } \\
\text { support of mental } \\
\text { health programs and }\end{array}$ \\
\hline
\end{tabular}




\begin{tabular}{|c|c|c|c|c|c|}
\hline $\begin{array}{r}\text { WA } \\
\text { PROGRAM AREA: Admir }\end{array}$ & $\begin{array}{l}\text { SHINGTON COUNTY MENTAL } \\
\text { istration }\end{array}$ & HEALTH PRC & AM: END 0 & F YEAR EVALUA & ATION \\
\hline Service Element & Objective & $\begin{array}{l}12 \text { Month } \\
\text { Target }\end{array}$ & $\begin{array}{l}12 \text { Month } \\
\text { Data }\end{array}$ & $\begin{array}{l}\% \text { Objective } \\
\text { Achieved }\end{array}$ & Comment \\
\hline $\begin{array}{l}\text { Administrative Ser- } \\
\text { vices } \\
\text { (cont.) }\end{array}$ & \begin{tabular}{|l|} 
To Assist Board of \\
Commissioners in mak- \\
ing policy decisions \\
regarding mental \\
health programs \\
(cont.) \\
To assure continued \\
and smooth operation \\
of county mental \\
health services \\
\\
\\
To assure the effec- \\
tive operation of \\
mental health ser- \\
vices provided by \\
county core staff
\end{tabular} & & 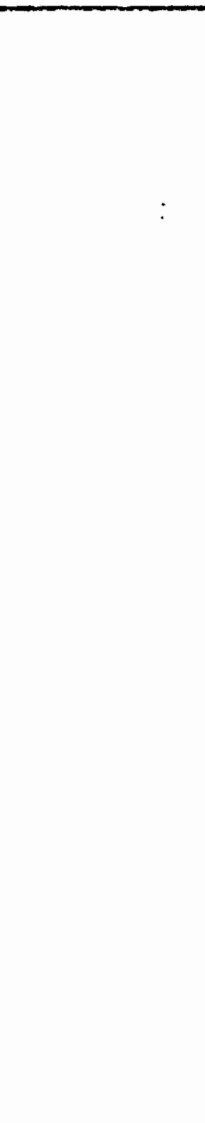 & & $\begin{array}{l}\text { actions demonstrate } \\
\text { satisfaction of this } \\
\text { objective. } \\
\\
\text { Fiscal matters were } \\
\text { handled on an accept- } \\
\text { able time frame. Ex- } \\
\text { ternal time lines were } \\
\text { adhered to throughout } \\
\text { the year in critical } \\
\text { areas. } \\
\text { Mental health core } \\
\text { staff positions were } \\
\text { managed well in com- } \\
\text { pleting their objec- } \\
\text { tives. Core staff de- } \\
\text { veloped systems to de- } \\
\text { liver services effec- } \\
\text { tively and to coordi- } \\
\text { nate client care and } \\
\text { county-wide service } \\
\text { delivery. }\end{array}$ \\
\hline
\end{tabular}


WASHINGTON COUNTY MENTAL HEALTH PROGRAM: END OF YEAR EVALUATION

PROGRAM AREA: Administration

\begin{tabular}{|c|c|c|c|c|c|}
\hline Service Element & Objective & $\begin{array}{l}12 \text { Month } \\
\text { Target }\end{array}$ & $\begin{array}{c}12 \text { Month } \\
\text { Date }\end{array}$ & $\begin{array}{l}\% \text { Objective } \\
\text { Achieved }\end{array}$ & Comment \\
\hline $\begin{array}{l}\text { Administrative Ser- } \\
\text { vices } \\
\text { (cont.) }\end{array}$ & $\begin{array}{l}\text { To gain community } \\
\text { confidence that coun- } \\
\text { ty efforts to develop } \\
\text { mental health ser- } \\
\text { vices will encompass } \\
\text { all program areas-- } \\
\text { MED, DD, A\&D }\end{array}$ & & & & $\begin{array}{l}\text { Advisory boards satis- } \\
\text { faction with mental } \\
\text { health service develop- } \\
\text { ment was demonstrated. } \\
\text { Washington County } \\
\text { Assn. for Retarded Cit- } \\
\text { izens supported the in- } \\
\text { creased resources allo- } \\
\text { cated to MR/DD Services } \\
\text { Coordination and agen- } \\
\text { cies. The Substance } \\
\text { Abuse Council approved } \\
\text { development of services } \\
\text { in A\&D for children, } \\
\text { Chicanos, American In- } \\
\text { dians, and those in- } \\
\text { volved with the crimi- } \\
\text { nal justice system. } \\
\text { Mental and Emotional } \\
\text { Health worked to in- } \\
\text { crease day treatment, } \\
\text { after care, prevention } \\
\text { and education re- } \\
\text { sources. }\end{array}$ \\
\hline
\end{tabular}


WASHINGTON COUNTY MENTAL HEALTH PROGRAM: END OF YEAR EVALUATION

PROGRAM AREA: Alcohol

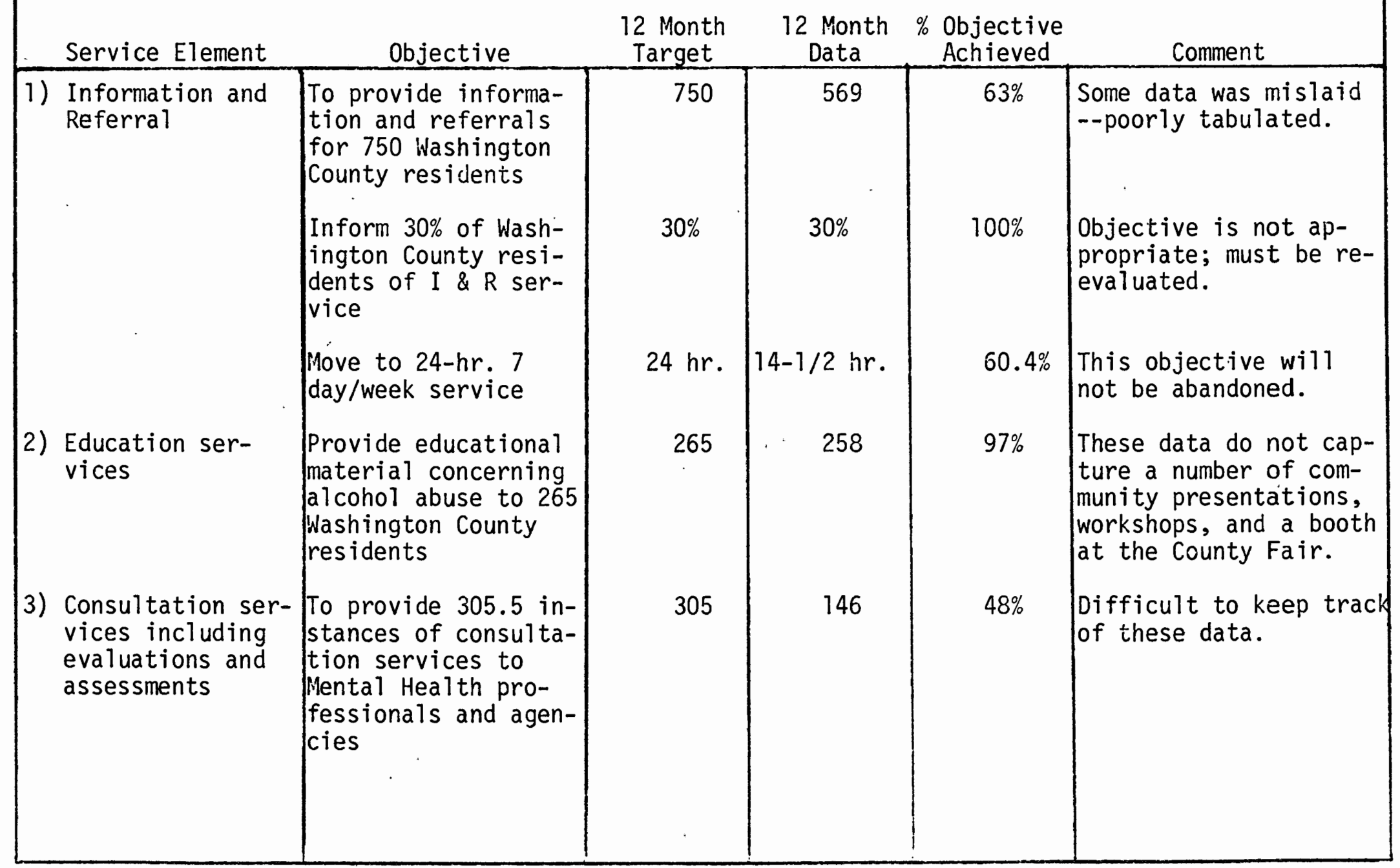




\section{WASHINGTON COUNTY MENTAL HEALTH PROGRAM: END OF YEAR EVALUATION}

PROGRAM AREA: Al cohol

\begin{tabular}{|c|c|c|c|c|c|c|}
\hline & Service Element & Objective & $\begin{array}{l}12 \text { Month } \\
\text { Target }\end{array}$ & $\begin{array}{l}12 \text { Month } \\
\text { Date }\end{array}$ & $\begin{array}{l}\% \text { Objective } \\
\text { Achieved } \\
\end{array}$ & Comment \\
\hline 4). & $\begin{array}{l}\text { Medication and } \\
\text { medical attention }\end{array}$ & $\begin{array}{l}\text { To provide } 645 \text { anta- } \\
\text { buse doses and } \\
36 \text { physicals for } \\
\text { Washington County } \\
\text { residents }\end{array}$ & 645 & 340 & $\begin{array}{l}52 \% \\
103 \%\end{array}$ & $\begin{array}{l}\text { Objective was not met } \\
\text { due to lack of contract } \\
\text { funds. } \\
\text { objective is appropri- } \\
\text { ate. }\end{array}$ \\
\hline 5) & $\begin{array}{l}\text { Outpatient ser- } \\
\text { vices }\end{array}$ & $\begin{array}{l}\text { To provide outpatient } \\
\text { counsel ing services } \\
\text { to } 615 \text { Washington } \\
\text { County residents }\end{array}$ & 615 & 329 & $54 \%$ & $\begin{array}{l}\text { Some subcontracted } \\
\text { agencies exceeded thein } \\
\text { objectives in this area } \\
\text { while others failed } \\
\text { dramatically. An ef- } \\
\text { fort will be made to } \\
\text { insure realistic objec- } \\
\text { tives for } 1977-1978 \text {. }\end{array}$ \\
\hline 6) & $\begin{array}{l}\text { Outreach/Advocacy } \\
\text { Services }\end{array}$ & $\begin{array}{l}\text { To provide outreach } \\
\text { and client advocacy } \\
\text { services to } 365 \\
\text { Washington County } \\
\text { residents }\end{array}$ & 365 & 330 & $90 \%$ & $\begin{array}{l}\text { Increase in visibility } \\
\text { and availability of } \\
\text { services. Not avail- } \\
\text { able are data reflect- } \\
\text { ing the impact of } 26 \\
\text { meetings relating to } \\
\text { outreach and advocacy. }\end{array}$ \\
\hline 7) & $\begin{array}{l}\text { Inservice Train- } \\
\text { ing }\end{array}$ & $\begin{array}{l}\text { To provide inservice } \\
\text { training to } 19 \text { Wash- } \\
\text { ington County Menta] } \\
\text { Health staff and } \\
\text { lagencies }\end{array}$ & 19 & 14 & $75 \%$ & $\begin{array}{l}\text { objective is appropri- } \\
\text { ate. }\end{array}$ \\
\hline
\end{tabular}


WASHINGTON COUNTY MENTAL HEALTH PROGRAM: END OF YEAR EVALUATION

PROGRAM AREA: Al coho

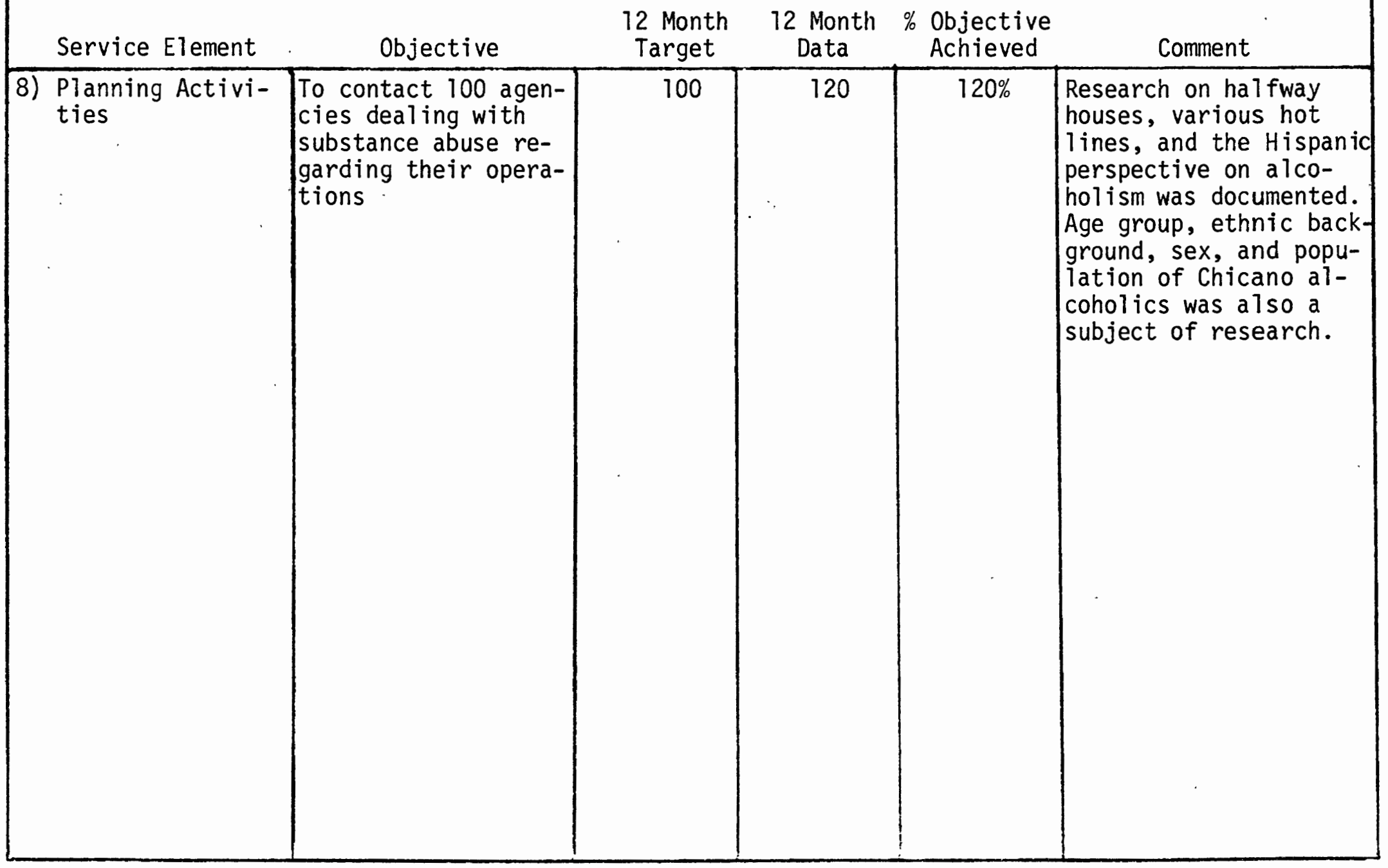

N 


\begin{tabular}{|c|c|c|c|c|c|}
\hline \multicolumn{6}{|c|}{$\begin{array}{l}\text { WASHINGTON COUNTY MENTAL HEALTH PROGRAM: END OF YEAR EVALUATION } \\
\text { PROGRAM AREA: ATcohol }\end{array}$} \\
\hline Alcohol Specialist & $\begin{array}{l}\text { Maintain an invento- } \\
\text { ry of services for } \\
\text { persons with alcohol } \\
\text { related services } \\
\text { Provide } 200 \text { units of } \\
\text { information referral } \\
\text { Provide inservice } \\
\text { training for } 4 \text { agen- } \\
\text { cies } \\
\text { Develop the Substance } \\
\text { Abuse component of } \\
\text { the } 1977-78 \text { Mental } \\
\text { Health Program } \\
\text { Provide staff ser- } \\
\text { vices to the Sub- } \\
\text { stance Abuse Council } \\
\text { Insure proper refer- } \\
\text { ral of clients to } \\
\text { agencies providing } \\
\text { service to substance } \\
\text { abusers }\end{array}$ & -- & $\begin{array}{r}223 \\
3 \\
\text { Done } \\
13 \text { meetings }\end{array}$ & $100 \%$ & $\begin{array}{l}\text { Not measurable or par- } \\
\text { ticularly relevant } \\
\text { Need to separate out } \\
\text { what "unit" means } \\
\text { persons vs. agencies. } \\
\text { Training was done by } \\
\text { by specialist in two } \\
\text { cases and purchased in } \\
\text { the other. } \\
\text { Again a major responsi- } \\
\text { bility but not written } \\
\text { measurably. } \\
\text { Needs to be changed to } \\
\text { be measurable. } \\
\text { This is basically an } \\
\text { I/R function and no } \\
\text { records were kept to } \\
\text { reflect this activity. }\end{array}$ \\
\hline
\end{tabular}


WASHINGTON COUNTY MENTAL HEALTH PROGRAM: END OF YEAR EVALUATION

PROGRAM AREA: Alcohol

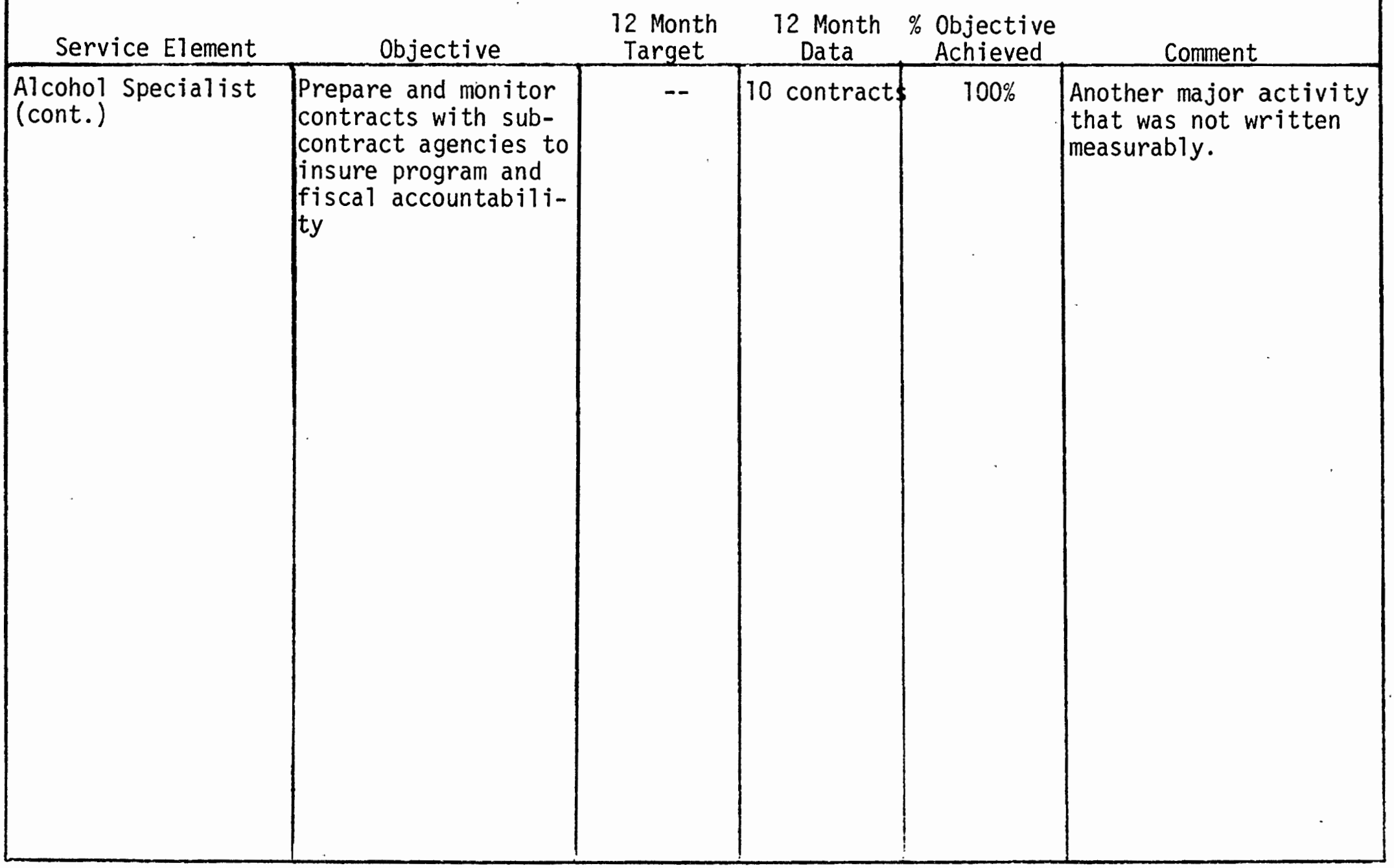




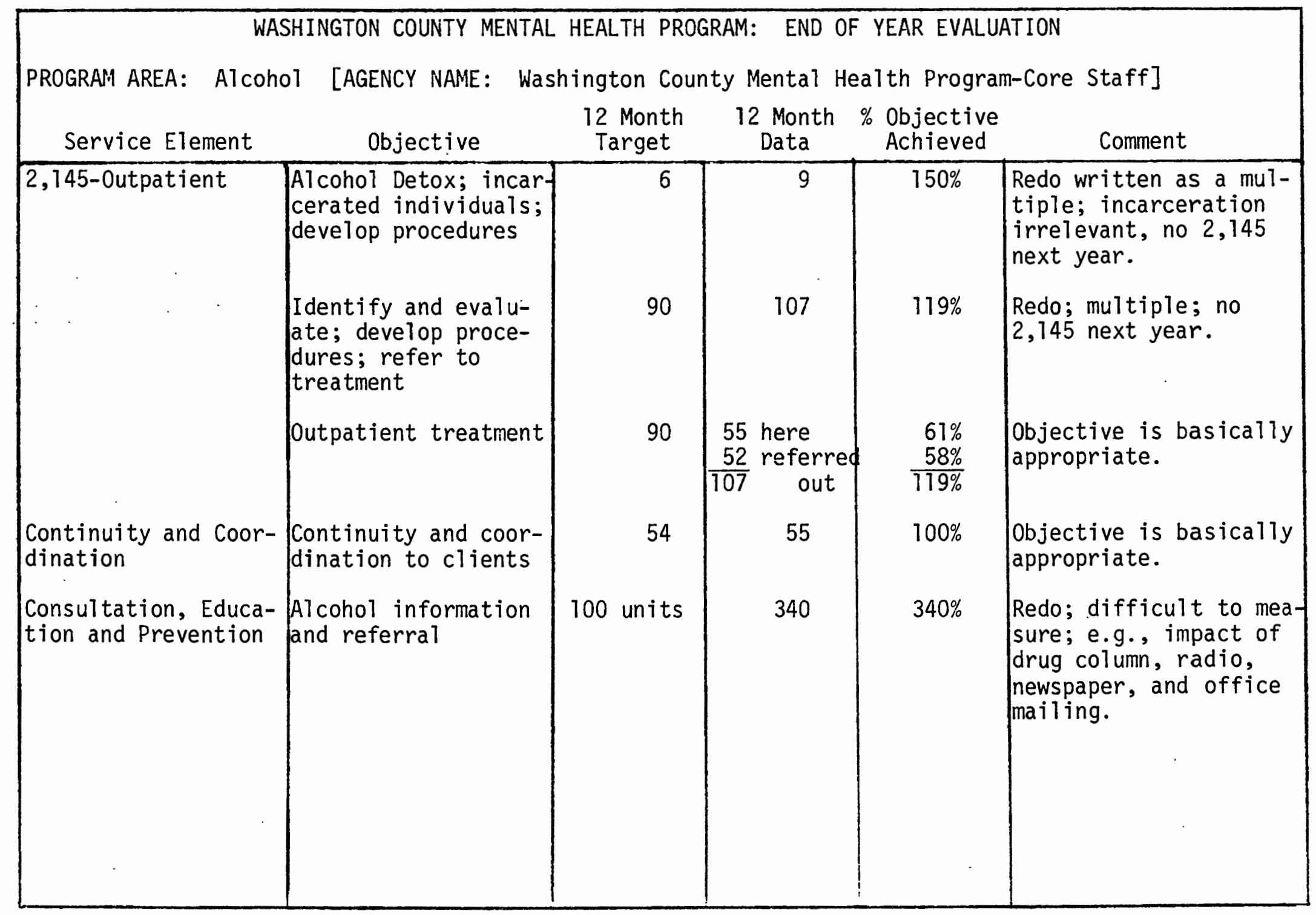




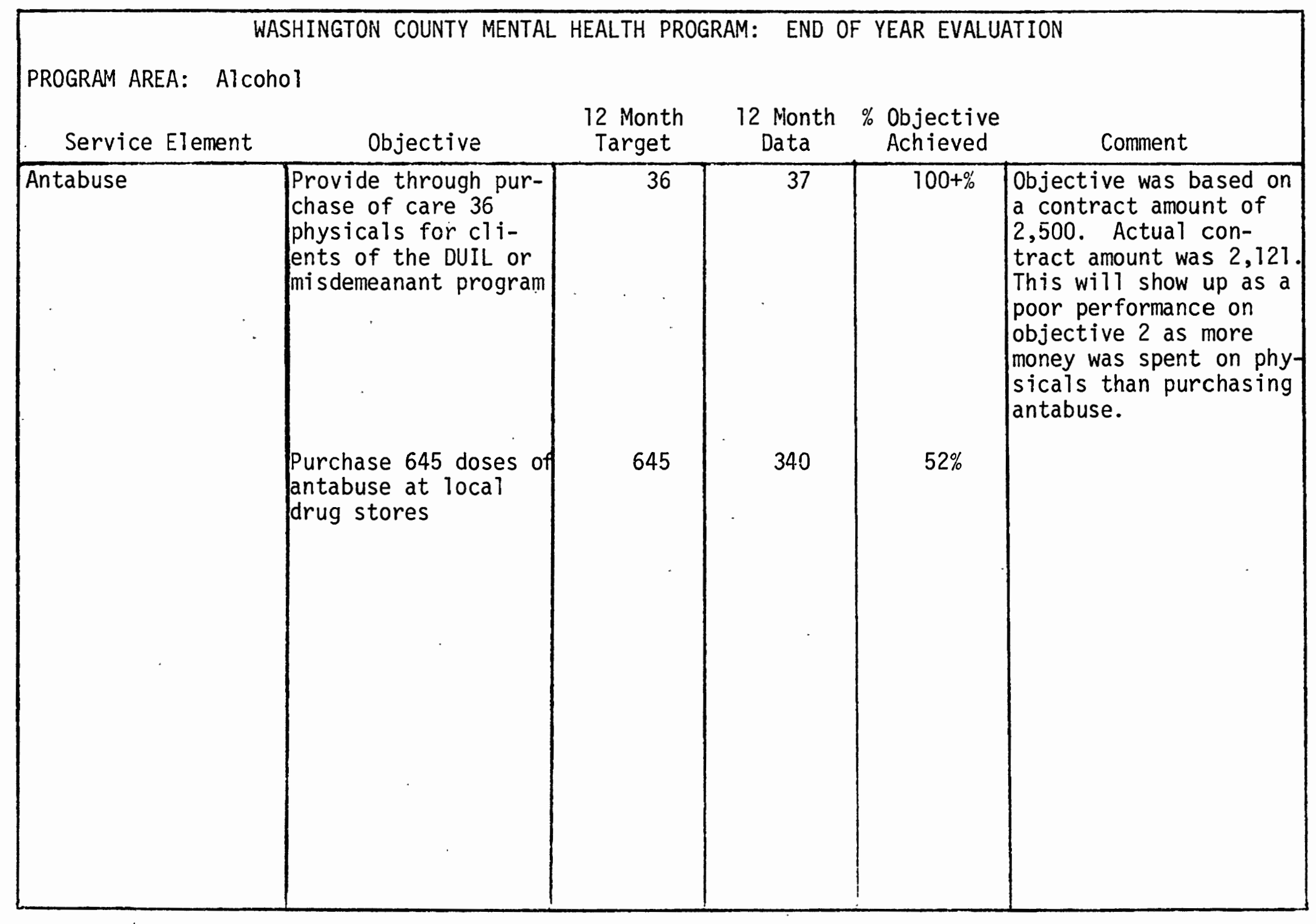




\begin{tabular}{|l|l|c|c|c|c|}
\hline \multicolumn{2}{|c|}{ WASHINGTON COUNTY MENTAL HEALTH PROGRAM: } \\
PROGRAM AREA: Alcohol \\
Service Element
\end{tabular}


WASHINGTON COUNTY MENTAL HEALTH PROGRAM: END OF YEAR EVALUATION

PROGRAM AREA: Alcohol

\begin{tabular}{|c|c|c|c|c|c|}
\hline Service Element & Objective & $\begin{array}{l}12 \text { Month } \\
\text { Target }\end{array}$ & $\begin{array}{l}12 \text { Month } \\
\text { Data }\end{array}$ & $\begin{array}{l}\% \text { Objective } \\
\text { Achieved }\end{array}$ & Comment \\
\hline $\begin{array}{l}\text { Substance Abuse } \\
\text { Council } \\
\text { (cont.) }\end{array}$ & $\begin{array}{l}\text { Provide the general } \\
\text { public with sub- } \\
\text { stance abuse infor- } \\
\text { mation and treatment } \\
\text { services } \\
\text { (cont.) } \\
\text { d) Give } 30 \text { presenta- } \\
\text { tions to civic, } \\
\text { service, or other } \\
\text { groups about sub- } \\
\text { stance abuse } \\
\text { Improve Council mem- } \\
\text { pers' knowledge of } \\
\text { substance abuse by } \\
\text { sponsoring } 10 \text { per- } \\
\text { sons for training }\end{array}$ & $\begin{array}{l}30 \\
10\end{array}$ & 13 & $\begin{array}{l}43 \% \\
60 \%\end{array}$ & $\begin{array}{l}\text { Not realistic due to } \\
\text { professional make-up } \\
\text { of the Council. }\end{array}$ \\
\hline
\end{tabular}


WASHINGTON COUNTY MENTAL HEALTH PROGRAM: END OF YEAR EVALUATION

PROGRAM AREA: Alcohol [AGENCY NAME: Centro Cultural]

\begin{tabular}{|c|c|c|c|c|c|}
\hline Service Element & Objective & $\begin{array}{l}12 \text { Month } \\
\text { Target }\end{array}$ & $\begin{array}{l}12 \text { Month } \\
\text { Data }\end{array}$ & $\begin{array}{l}\% \text { Objective } \\
\text { Achieved }\end{array}$ & Comment \\
\hline $\begin{array}{l}\text { Alcohol Counseling } \\
\text { Centro }\end{array}$ & $\begin{array}{l}\text { Provided } 151 \text { units } \\
\text { of information re- } \\
\text { ferral }\end{array}$ & 300 & 151 & $50 \%$ & None \\
\hline \multirow[t]{4}{*}{$:$} & $\begin{array}{l}\text { Provided private and } \\
\text { family counseling to } \\
40 \text { caseload of cli- } \\
\text { ents }\end{array}$ & 150 & 40 & $26.6 \%$ & $\begin{array}{l}\text { Caseload will increase } \\
\text { gradually; } 80 \text { units is } \\
\text { a more realistic goal. }\end{array}$ \\
\hline & $\begin{array}{l}\text { Provided } 70 \text { units of } \\
\text { advocacy and liaison } \\
\text { for the Chicano com- } \\
\text { munity }\end{array}$ & 100 & 70 & $70 \%$ & None \\
\hline & $\begin{array}{l}\text { Provided outreach } \\
\text { services to } 90 \text { indi- } \\
\text { viduals. This activ- } \\
\text { ity consisted basi- } \\
\text { cally of camp and/or } \\
\text { home visits. } 140 \\
\text { units of encounters. }\end{array}$ & 100 & 90 & $90 \%$ & $\begin{array}{l}\text { This type of activity } \\
\text { was productive in the } \\
\text { sense that it increased } \\
\text { the visibility and } \\
\text { credibility of progress }\end{array}$ \\
\hline & $\begin{array}{l}\text { Developed data base } \\
\text { for alcohol services } \\
\text { a) contacted } 120 \\
\text { agencies that } \\
\text { deal with sub- } \\
\text { stance abuse } \\
\text { problems }\end{array}$ & 100 & 120 & $100 \%$ & $\begin{array}{l}\text { Research on halfway } \\
\text { houses, various hot } \\
\text { lines, and the Hispanic } \\
\text { perspective on alcohol } \\
\text { ism was documented. } \\
\text { Age group, ethnic back- } \\
\text { ground, sex and }\end{array}$ \\
\hline
\end{tabular}




\section{WASHINGTON COUNTY MENTAL HEALTH PROGRAM: END OF YEAR EVALUATION}

PROGRAM AREA: Alcohol [AGENCY NAME: Centro Cultural]

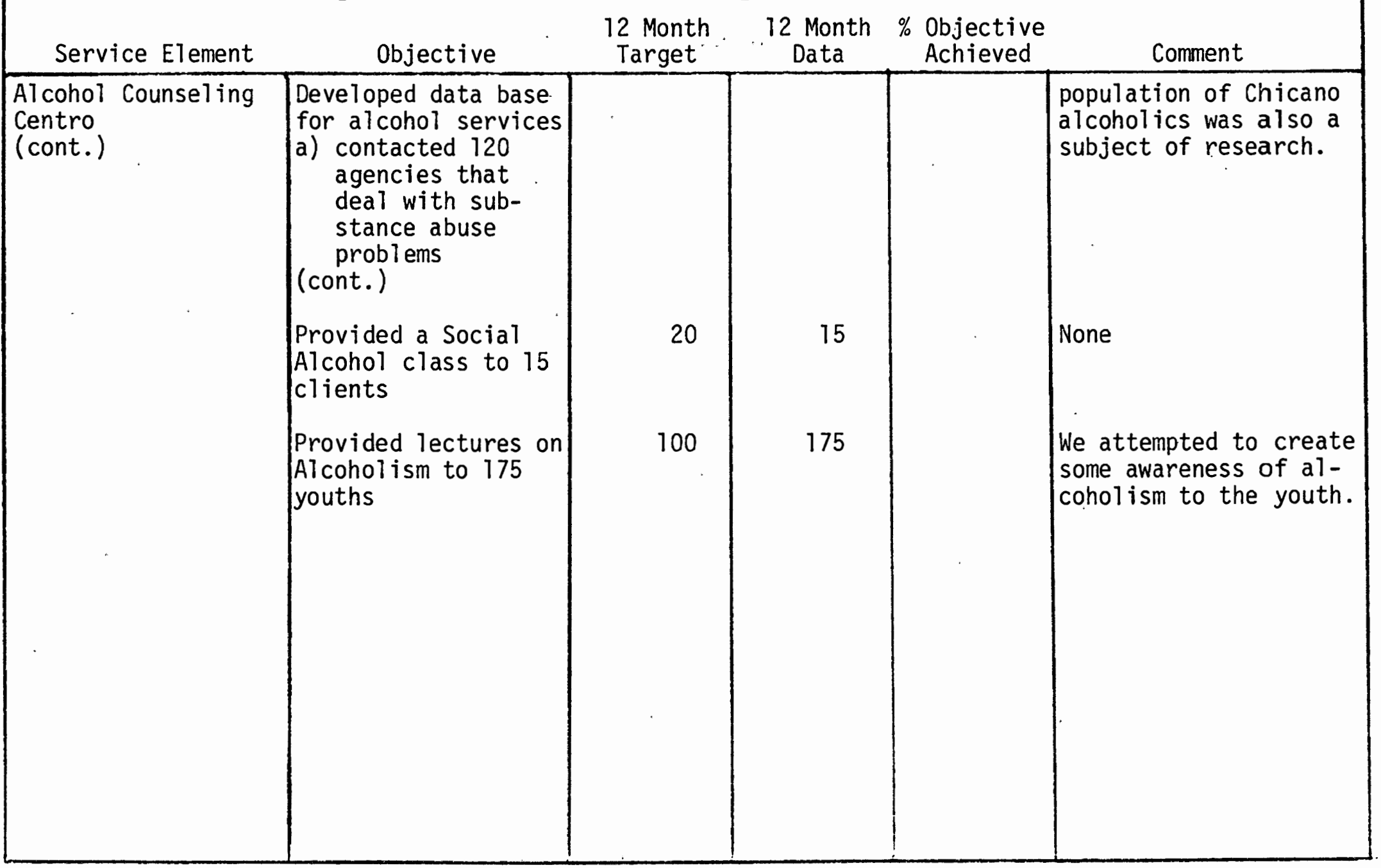


WASHINGTON COUNTY MENTAL HEALTH PROGRAM: END OF YEAR EVALUATION

PROGRAM AREA: Alcohol [AGENCY NAME: Washington County Community Corrections]

\begin{tabular}{|c|c|c|c|c|c|}
\hline Service Element & Objective & $\begin{array}{l}12 \text { Month } \\
\text { Target }\end{array}$ & $\begin{array}{l}12 \text { Month } \\
\text { Date }\end{array}$ & $\begin{array}{l}\text { \% Objective } \\
\text { Achieved }\end{array}$ & Comment \\
\hline \multirow[t]{3}{*}{$\begin{array}{l}\text { Washington County } \\
\text { Community Correc- } \\
\text { tions. }\end{array}$} & $\begin{array}{l}\text { Contract with mental } \\
\text { health professionals } \\
\text { to provide } 10 \text { full } \\
\text { psychological as- } \\
\text { sessments }\end{array}$ & $\begin{array}{l}7.5 \text { psych. } \\
\text { evaluations }\end{array}$ & $\begin{array}{l}9 \text { psych. } \\
\text { evaluations }\end{array}$ & $120 \%$ & $\begin{array}{l}\text { The original objec- } \\
\text { tives of the grant are } \\
\text { pro-rated for } 9 \text { months } \\
\text { as grant activities, } \\
\text { were not initiated un- } \\
\text { til october } 1976 \text {. }\end{array}$ \\
\hline & $\begin{array}{l}\text { Provide diagnostic } \\
\text { evaluations and as- } \\
\text { sessments to } 100 \\
\text { program clients }\end{array}$ & $\begin{array}{l}75 \text { diagnos- } \\
\text { tic evalua- } \\
\text { tions }\end{array}$ & $\mid \begin{array}{l}50 \text { diagnos- } \\
\text { tic evalua- } \\
\text { tions }\end{array}$ & $67 \%$ & $\begin{array}{l}\text { We expected to sched- } \\
\text { ule } 4 \text { staffings every } \\
\text { other week throughout } \\
\text { the year; therefore we } \\
\text { speculated we could } \\
\text { evaluate } 100 \text { people. } \\
\text { Due to cancellations } \\
\text { and no-shows we were } \\
\text { unable to meet this } \\
\text { objective. However, } \\
10 \text { people were re- } \\
\text { staffed; therefore, } \\
\text { although } 50 \text { evaluations } \\
\text { were completed, 60 } \\
\text { staffings were held. }\end{array}$ \\
\hline & $\begin{array}{l}\text { Provide alcohol } \\
\text { counseling and re- } \\
\text { ferral services to } \\
50 \text { identified alco- } \\
\text { hol abusers }\end{array}$ & $\begin{array}{l}\text { Referral } \\
\text { and coun- } \\
\text { sel ing of } \\
37.5 \\
\text { abusers }\end{array}$ & 50 & $133 \%$ & $\begin{array}{l}\text { Seven staff members } \\
\text { and two volunteers con- } \\
\text { ducted staffings with } \\
\text { John Hughes on a regu- } \\
\text { lar basis. Additional }\end{array}$ \\
\hline
\end{tabular}


WASHINGTON COUNTY MENTAL HEALTH PROGRAM: END OF YEAR EVALUATION

PROGRAM AREA: Alcohol [AGENCY NAME: Washington County Community Corrections]

\begin{tabular}{|c|c|c|c|c|c|}
\hline Service Element & Objective & $\begin{array}{c}12 \text { Month } \\
\text { Target }\end{array}$ & $\begin{array}{l}12 \text { Month } \\
\text { Data }\end{array}$ & $\begin{array}{l}\% \text { Objective } \\
\text { Achieved }\end{array}$ & Comment \\
\hline $\begin{array}{l}\text { Washington County } \\
\text { Community Correc- } \\
\text { tions } \\
\text { (cont.) } \\
\vdots\end{array}$ & $\begin{array}{l}\text { Provide alcohol } \\
\text { counseling and re- } \\
\text { ferral services to } \\
50 \text { identified alco- } \\
\text { hol abusers } \\
\text { (cont.) } \\
\\
\text { Provide supportive } \\
\text { counseling to } 50 \\
\text { program clients }\end{array}$ & $\begin{array}{l}\text { Supportive } \\
\text { counseling } \\
\text { of } 37.5 \\
\text { clients }\end{array}$ & $\begin{array}{l}22 \text { group } \\
\text { members }\end{array}$ & $59 \%$ & $\begin{array}{l}\text { traning time of two } \\
\text { hours per month was } \\
\text { scheduled. We also } \\
\text { utilized staffing time } \\
\text { that was available due } \\
\text { to a no-show for } \\
\text { training. This train- } \\
\text { ing time assisted staff } \\
\text { in working with cli- } \\
\text { ents who were evalu- } \\
\text { ated as well as the } \\
\text { balance of their case- } \\
\text { load. } \\
\text { Two volunteers at- } \\
\text { tended the bi-weekly } \\
\text { night group on a regu- } \\
\text { lar basis. Twenty- } \\
\text { two people were in- } \\
\text { volved in the group } \\
\text { over the past } 9 \text { months } \\
\text { and several are con- } \\
\text { tinuing. The average } \\
\text { number of people at } \\
\text { group was } 11 \text { and the } \\
\text { group met for approxi- } \\
\text { mately } 40 \text { hours. }\end{array}$ \\
\hline
\end{tabular}




\section{WASHINGTON COUNTY MENTAL HEALTH PROGRAM: END OF YEAR EVALUATION}

PROGRAM AREA: Alcohol [AGENCY NAME: Native American Rehabilitation Assn.]

\begin{tabular}{|c|c|c|c|c|c|}
\hline Service Element & Objective & $\begin{array}{l}12 \text { Month } \\
\text { Target }\end{array}$ & $\begin{array}{l}12 \text { Month } \\
\text { Data }\end{array}$ & $\begin{array}{c}\% \text { Objective } \\
\text { Achieved }\end{array}$ & Comment \\
\hline $\begin{array}{l}\text { Washington County } \\
\text { Outreach }\end{array}$ & $\begin{array}{l}\text { Locate and identify } \\
120 \text { Native American } \\
\text { persons from Wash- } \\
\text { ington County and in } \\
\text { need of NARA ser- } \\
\text { vices over a } 12 \\
\text { month period } \\
\text { Provide initial con- } \\
\text { tact with } 75 \text { of the } \\
\text { target population } \\
\text { and initiate treat- } \\
\text { ment } \\
\text { Develop treatment } \\
\text { plans and involve in } \\
\text { treatment } 50 \text { Native } \\
\text { American persons } \\
\text { from Washington } \\
\text { County } \\
\text { Assist the Washing- } \\
\text { ton County Alcohol } \\
\text { Specialist. in pro- } \\
\text { gram planning by } \\
\text { having monthly meet- } \\
\text { ings with outreach } \\
\text { worker }\end{array}$ & $\begin{array}{l}75 \\
50\end{array}$ & $\begin{array}{c}79 \text { individ. } \\
9 \text { families } \\
30 \\
37 \\
18 \text { Mtgs. }\end{array}$ & $\begin{array}{l}100 \% \\
40 \% \\
74 \% \\
100+\%\end{array}$ & $\begin{array}{l}\text { } \\
\text { More people were pro- } \\
\text { vided with initial con- } \\
\text { tacts but the data sys- } \\
\text { tem only reflects this } \\
\text { number. }\end{array}$ \\
\hline
\end{tabular}




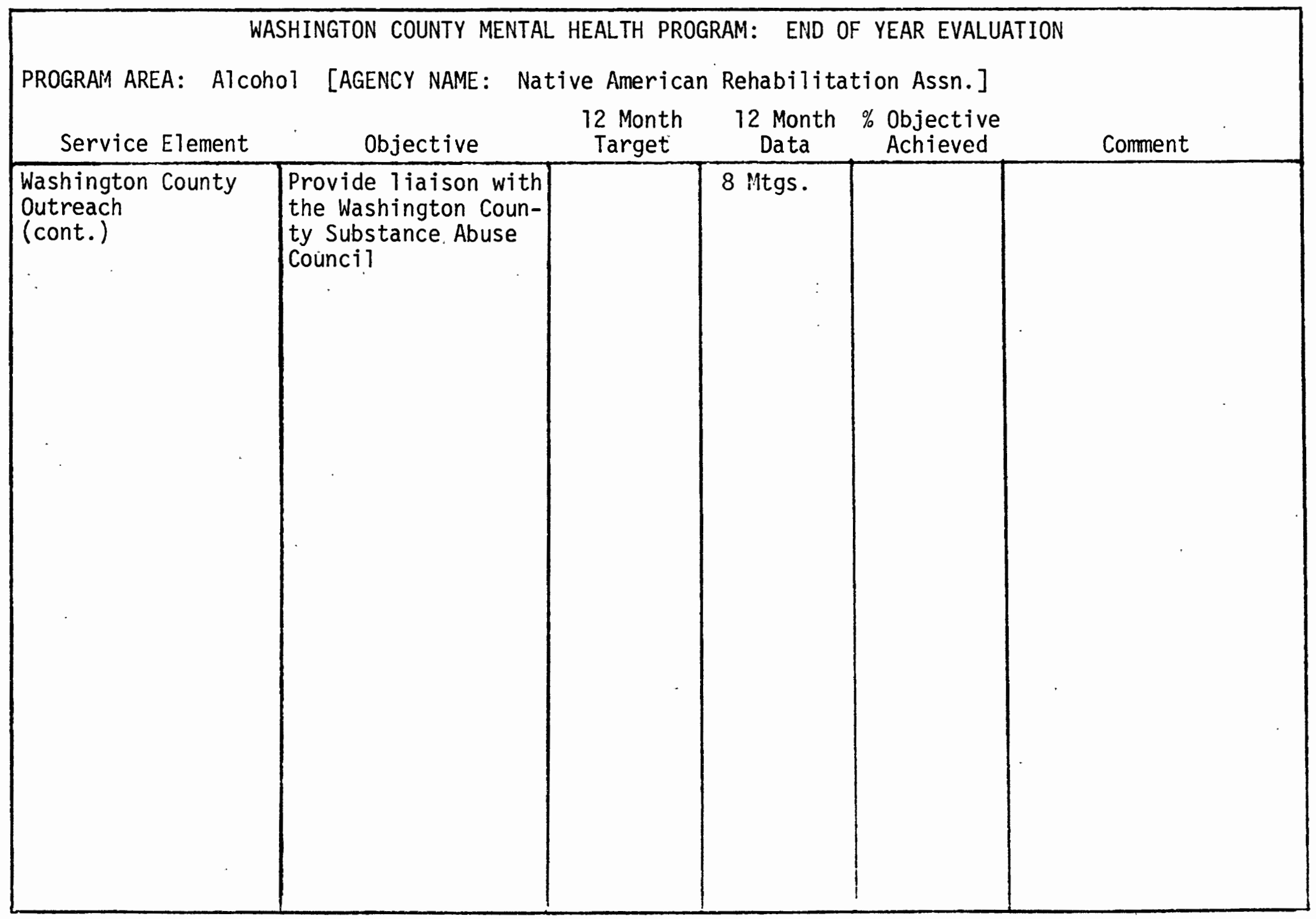

$\stackrel{\omega}{\not}$ 


\begin{tabular}{|c|c|c|c|c|c|}
\hline \multicolumn{6}{|c|}{$\begin{array}{l}\text { WASHINGTON COUNTY MENTAL HEALTH PROGRAM: END OF YEAR EVALUATION } \\
\text { PROGRAM AREA: Alcohol [AGENCY NAME: Tri-County Community Counci1] }\end{array}$} \\
\hline Service Element & Objective & $\begin{array}{l}12 \text { Month } \\
\text { Target }\end{array}$ & $\begin{array}{l}12 \text { Month } \\
\text { Data }\end{array}$ & $\begin{array}{l}\% \text { Objective } \\
\text { Achieved }\end{array}$ & Comment \\
\hline \multirow[t]{4}{*}{$\begin{array}{l}\text { Alcohol and Drug } \\
\text { Treatment }\end{array}$} & $\begin{array}{l}\text { Identify and record } \\
200 \text { calls requesting } \\
\text { information concern- } \\
\text { ing alcohol abuse. } \\
\text { Document referrals } \\
\text { made and gaps in } \\
\text { service discovered }\end{array}$ & 200 & 20 & $10 \%$ & $\begin{array}{l}\text { See Item \#1 of memo } \\
\text { dated } 1 / 19 / 77 \text { (Exhibit } \\
\text { 2) }\end{array}$ \\
\hline & $\begin{array}{l}\text { Inform } 30 \% \text { of Wash- } \\
\text { ington County resi- } \\
\text { dents of the exis- } \\
\text { tence of the I\&R } \\
\text { Service }\end{array}$ & $30 \%$ & $30 \%$ & $100 \%$ & $\begin{array}{l}\text { See I tem } \# 2 \text { of memo } \\
\text { dated } 1 / 19 / 77 \text { (Exhibit } \\
\text { 2) }\end{array}$ \\
\hline & $\begin{array}{l}\text { Provide inservice } \\
\text { training to staff } \\
\text { and volunteers of } \\
\text { the I\&R Service }\end{array}$ & 5 & 5 & $100 \%$ & $\begin{array}{l}\text { See last paragraph of } \\
\text { page } 1 \text { of memo dated } \\
10 / 20 / 76 \text { (Exhibit } 5 \text { ) } \\
\text { and Item \#4 of memo } \\
\text { dated } 1 / 19 / 77 \text { (Exhibit } \\
\text { 2) }\end{array}$ \\
\hline & $\begin{array}{l}\text { Move toward provid- } \\
\text { ing I\&R Service on a } \\
\text { 24-hour, } 7 \text { day a } \\
\text { week basis }\end{array}$ & $24 / \mathrm{Hr}$. & $14-1 / 2 / \mathrm{Hr}$ & $60.4 \%$ & $\begin{array}{l}\text { See Item \#4 of memo } \\
\text { dated } 1 / 19 / 77 \text { (Exhibit } \\
\text { 2) }\end{array}$ \\
\hline
\end{tabular}




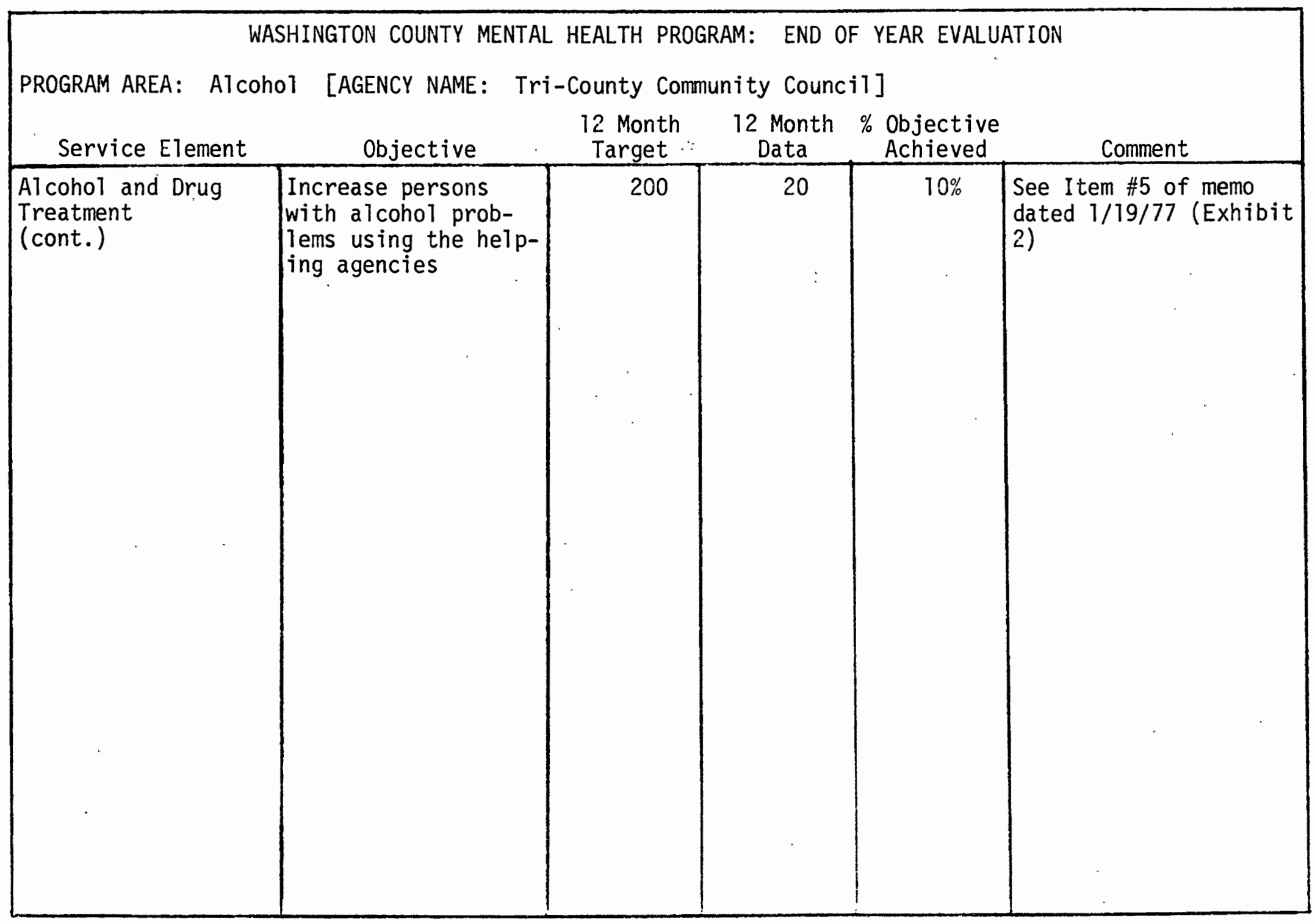

$\omega$ 


\section{WASHINGTON COUNTY MENTAL HEALTH PROGRAM: END OF YEAR EVALUATION}

PROGRAM AREA: Alcohol [AGENCY NAME: Tualatin Valley Mental Health Center]

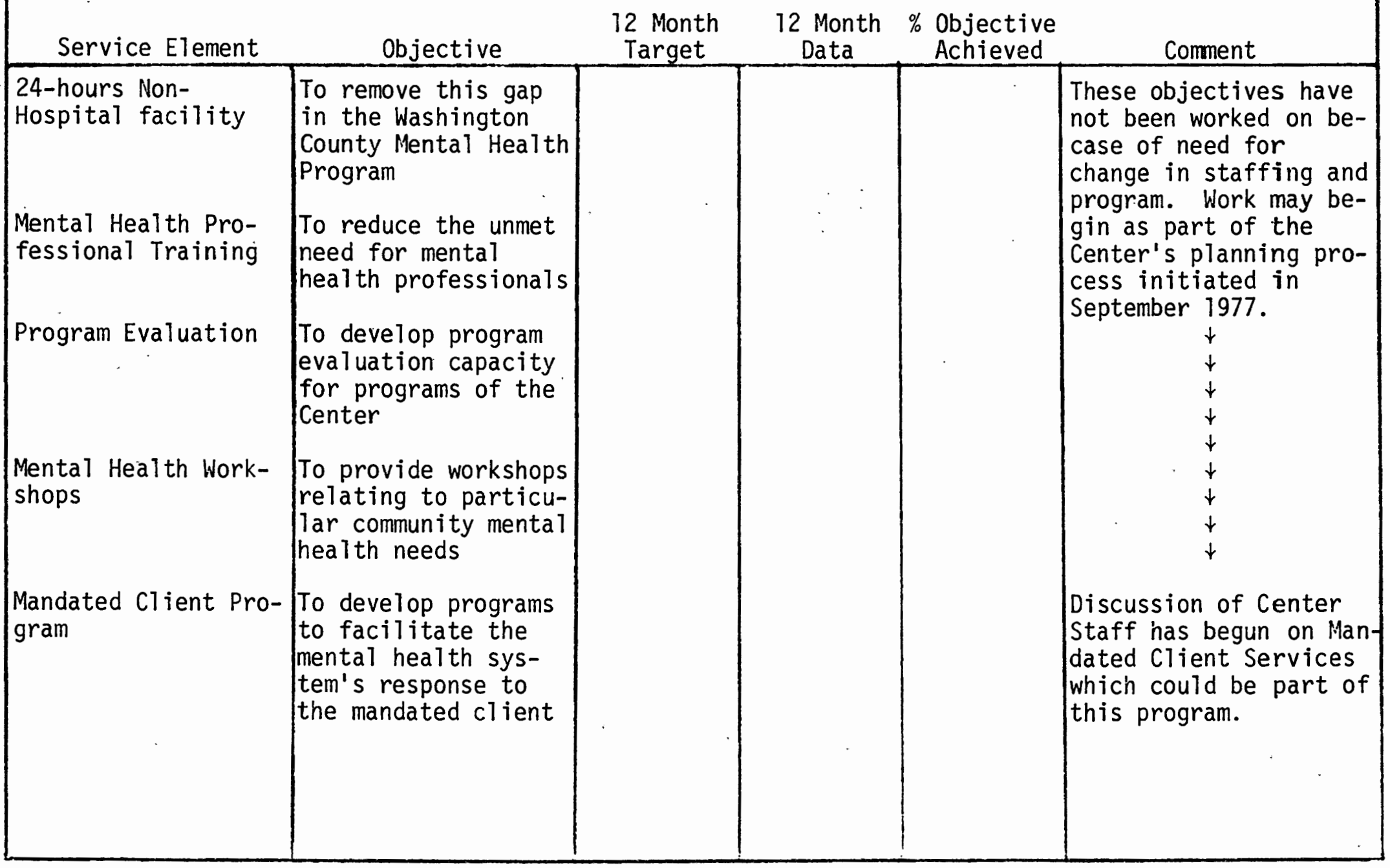




\section{WASHINGTON COUNTY MENTAL HEALTH PROGRAM: END OF YEAR EVALUATION}

PROGRAM AREA: Alcohol [AGENCY NAME: Tualatin Valley Mental Health Center]

\begin{tabular}{|c|c|c|c|c|c|}
\hline Service Element & Objective & $\begin{array}{l}12 \text { Month } \\
\text { Target. }\end{array}$ & $\begin{array}{l}12 \text { Month } \\
\text { Data }\end{array}$ & $\begin{array}{c}\% \text { Objective } \\
\text { Achieved }\end{array}$ & Comment \\
\hline $\begin{array}{l}\text { Mandated Client Pro- } \\
\text { gram } \\
\text { (cont.) }\end{array}$ & $\begin{array}{l}\text { Provide outpatient } \\
\text { services to } 200 \\
\text { alcohol-dependent } \\
\text { persons } \\
\text { Reduce the impact of } \\
\text { alcohol and related } \\
\text { problems on } 100 \text { cli- } \\
\text { ents } \\
\text { Provide } 50 \text { units of } \\
\text { information/referral } \\
\text { services } \\
\text { Increase availabili- } \\
\text { ty of services to } \\
\text { minority populations } \\
\text { in Washington County }\end{array}$ & \begin{tabular}{|r|}
200 \\
100 \\
50 \\
1 day/week
\end{tabular} & 130 & $23 \%$ & $\begin{array}{l}\text { We are under on the } \\
\text { service objective. } \\
\text { We are over on the re- } \\
\text { ducing impact objec- } \\
\text { tive. } \\
\text { We have achieved the } \\
\text { information objective. } \\
\text { This objective has } \\
\text { been achieved. }\end{array}$ \\
\hline
\end{tabular}




\section{WASHINGTON COUNTY MENTAL HEALTH PROGRAM: END OF YEAR EVALUATION}

PROGRAM AREA: Alcohol [AGENCY NAME: Washington County Youth Contact]

\begin{tabular}{|c|c|c|c|c|c|}
\hline Service Element & Objective & $\begin{array}{l}12 \text { Month } \\
\text { Target }\end{array}$ & $\begin{array}{l}12 \text { Month } \\
\text { Data }\end{array}$ & $\begin{array}{l}\text { \% Objective } \\
\text { Achieved }\end{array}$ & Comment \\
\hline \multirow[t]{2}{*}{ A7cohol Treatment } & $\begin{array}{l}\text { To evaluate } 200 \text { cli- } \\
\text { ents for acceptance } \\
\text { into the Youth Con- } \\
\text { tact program }\end{array}$ & 200 & $\ldots$ & $32 \%$ & $\begin{array}{l}\text { Case records reflect } \\
\text { client evaluations, } \\
\text { program acceptance } \\
\text { criteria, and feedback } \\
\text { reported to the refer- } \\
\text { ral agency. }\end{array}$ \\
\hline & $\begin{array}{l}\text { To serve } 150 \mathrm{clients} \\
\text { in the program }\end{array}$ & 150 & 50 & $33 \%$ & $\begin{array}{l}\text { Client attendance rec- } \\
\text { ords have been kept } \\
\text { which reflect both the } \\
\text { number of youth groups } \\
\text { held and individual } \\
\text { attendance at each. } \\
\text { The estimated number } \\
\text { of client evaluations } \\
\text { and clients served, as } \\
\text { stated in objectives } \\
\text { l and } 2 \text {, appear to have } \\
\text { been too high for the } \\
\text { following reasons: } \\
\text { (a) The hiring of } \\
\text { the Youth Contact Di- } \\
\text { rector was effective } \\
\text { on October l1, 1976, } \\
\text { approximately three and } \\
\text { one-half months into } \\
\text { the fiscal year, thus } \\
\text { curtailing directed }\end{array}$ \\
\hline
\end{tabular}


WASHINGTON COUNTY MENTAL HEALTH PROGRAM: END OF YEAR EVALUATION

PROGRAM AREA: Alcohol [AGENCY NAME: Washington County Youth Contact]

\begin{tabular}{|c|c|c|c|c|c|}
\hline Service Element & Objective & $\begin{array}{c}12 \text { Month } \\
\text { Target }\end{array}$ & $\begin{array}{l}12 \text { Month } \\
\text { Data }\end{array}$ & $\begin{array}{l}\% \text { Objective } \\
\text { Achieved }\end{array}$ & Comment \\
\hline $\begin{array}{l}\text { Alcohol Treatment } \\
\text { (cont.) }\end{array}$ & $\begin{array}{l}\text { To evaluate } 200 \text { cli- } \\
\text { ents for acceptance } \\
\text { into the Youth Con- } \\
\text { tact program } \\
\text { (cont.) } \\
\text { To serve } 150 \text { clients } \\
\text { in the program } \\
\text { (cont.) } \\
\\
\text { To serve } 75 \text { parents } \\
\text { of clients in the } \\
\text { program }\end{array}$ & 75 & 68 & $90 \%$ & $\begin{array}{l}\text { efforts toward client } \\
\text { recruitment and selec- } \\
\text { tion and county pro- } \\
\text { gram exposure to fa- } \\
\text { cilitate and increase } \\
\text { such. } \\
\text { (b) Limitations upon } \\
\text { the available agency } \\
\text { manpower to conduct } \\
\text { the projected number } \\
\text { of evaluations and to } \\
\text { provide ongoing treat- } \\
\text { ment to those served. } \\
\text { (c) Limitations on } \\
\text { the exposure of Youth } \\
\text { Contact to the county } \\
\text { at large. } \\
\text { (d) The voluntary re- } \\
\text { ferral nature of the } \\
\text { majority of Youth Con- } \\
\text { tact referrals. } \\
\text { Records have been kept } \\
\text { which reflect the num- } \\
\text { ber and the frequency } \\
\text { of parent groups and } \\
\text { the individual atten- } \\
\text { dance at each. }\end{array}$ \\
\hline
\end{tabular}




\section{WASHINGTON COUNTY MENTAL HEALTH PROGRAM: END OF YEAR EVALUATION}

PROGRAM AREA: Drug

\begin{tabular}{|c|c|c|c|c|c|c|}
\hline & Service Element & Objective & $\begin{array}{l}12 \text { Month } \\
\text { Target }\end{array}$ & $\begin{array}{l}12 \text { Month } \\
\text { Data }\end{array}$ & $\begin{array}{c}\% \text { Objective } \\
\text { Achieved }\end{array}$ & Comment \\
\hline 1) & $\begin{array}{l}\text { Information/ } \\
\text { Referral Services }\end{array}$ & $\begin{array}{l}\text { To provide informa- } \\
\text { tion and referral } \\
\text { services to } 200 \mathrm{cli}- \\
\text { ents }\end{array}$ & 200 & 641 & $321 \%$ & $\begin{array}{l}\text { Difficult to measure } \\
\text { impact of some of } \\
\text { these services. Also } \\
\text { difficult to separate } \\
\text { drug from alcohol in } \\
\text { this area. Will con- } \\
\text { sider adjusting this } \\
\text { objective to avoid } \\
\text { these problems. }\end{array}$ \\
\hline 2) & $\begin{array}{l}\text { Outpatient Ser- } \\
\text { vices }\end{array}$ & $\begin{array}{l}\text { To provide outpa- } \\
\text { tient counseling } \\
\text { services to } 140 \mathrm{cli-} \\
\text { ents }\end{array}$ & 140 & 170 & $120 \%$ & $\begin{array}{l}\text { Will tighten up and } \\
\text { separate components } \\
\text { of this objective for } \\
1977-78 \text { in an effort } \\
\text { to secure more valu- } \\
\text { able evaluation data. }\end{array}$ \\
\hline 3) & $\begin{array}{l}\text { Continuity and } \\
\text { Coordination of } \\
\text { Services }\end{array}$ & $\begin{array}{l}\text { To move } 144 \text { clients } \\
\text { smoothly through the } \\
\text { system }\end{array}$ & 144 & 148 & $103 \%$ & $\begin{array}{l}\text { Objective is appropri- } \\
\text { ate. }\end{array}$ \\
\hline 4) & Detoxification & $\begin{array}{l}\text { To provide detox } \\
\text { services to } 10 \mathrm{cli-} \\
\text { ents }\end{array}$ & 10 & 19 & $190 \%$ & $\begin{array}{l}\text { Objective will be in- } \\
\text { creased for } 1977-1978 \text {. }\end{array}$ \\
\hline 5) & Inservice Train- & $\begin{array}{l}\text { To provide inservice } \\
\text { training for } 3 \text { agen- } \\
\text { cies in Washington } \\
\text { County }\end{array}$ & 3 & 4 & $133 \%$ & $\begin{array}{l}\text { Objective needs to be } \\
\text { more specific for } \\
1977-1978 \text {. }\end{array}$ \\
\hline
\end{tabular}


WASHINGTON COUNTY MENTAL HEALTH PROGRAM: END OF YEAR EVALUATION

PROGRAM AREA: Drug

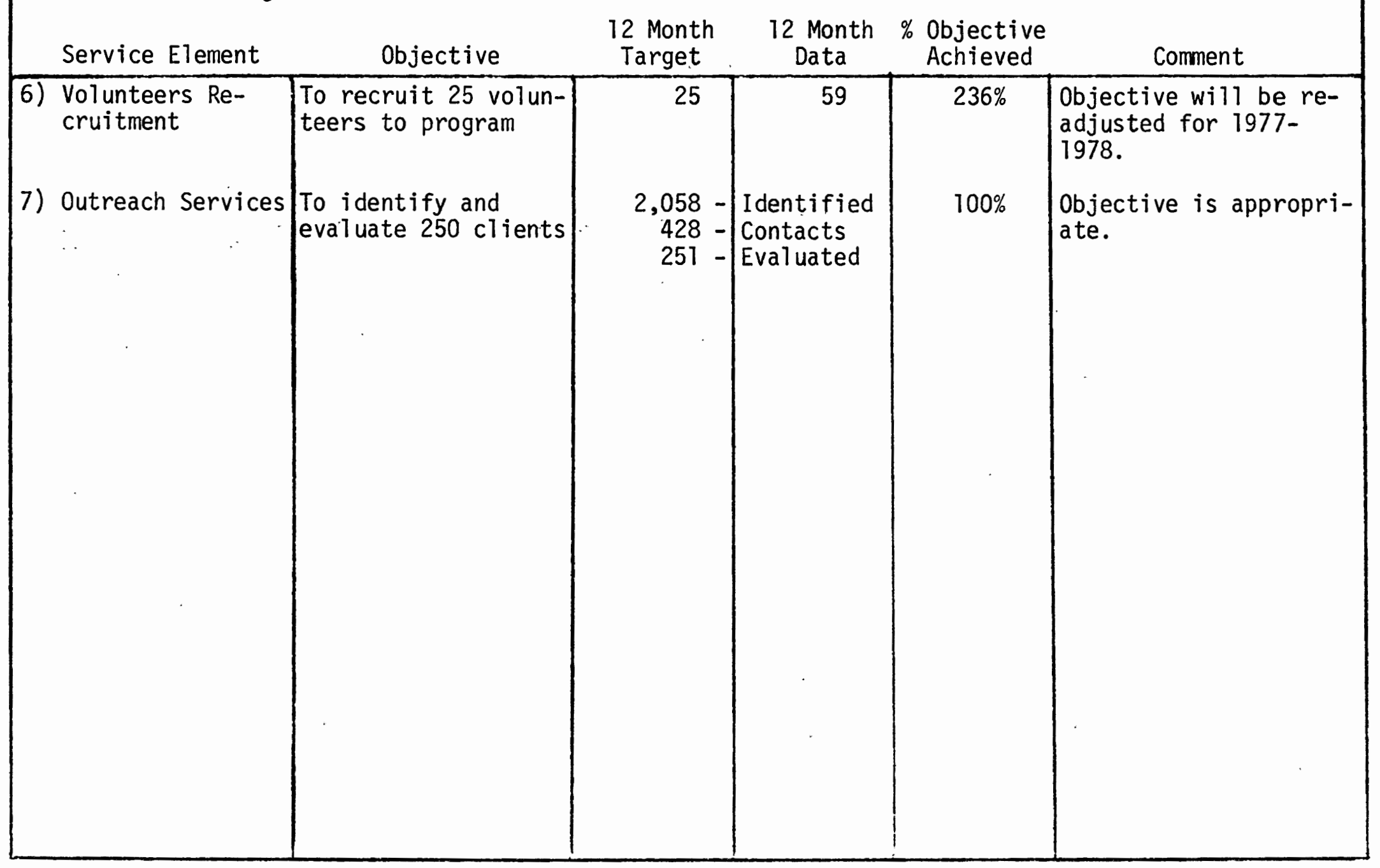

$\vec{n}$ 


\section{WASHINGTON COUNTY MENTAL HEALTH PROGRAM: END OF YEAR EVALUATION}

PROGRAM AREA: Drug

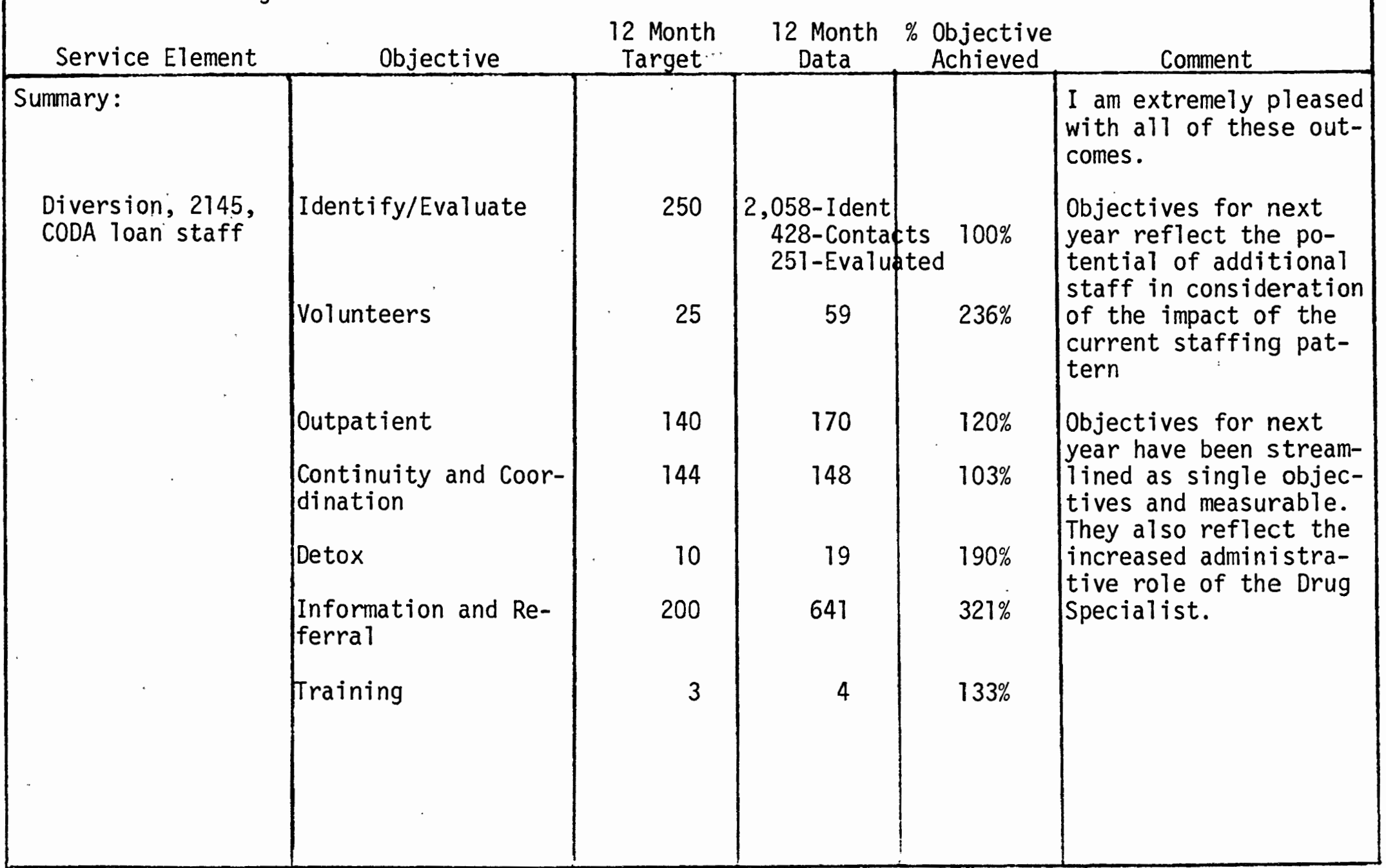




\section{WASHINGTON COUNTY MENTAL HEALTH PROGRAM: END OF YEAR EVALUATION}

PROGRAM AREA: Drug [AGENCY NAME: Washington County Mental Health Program--Core Staff]

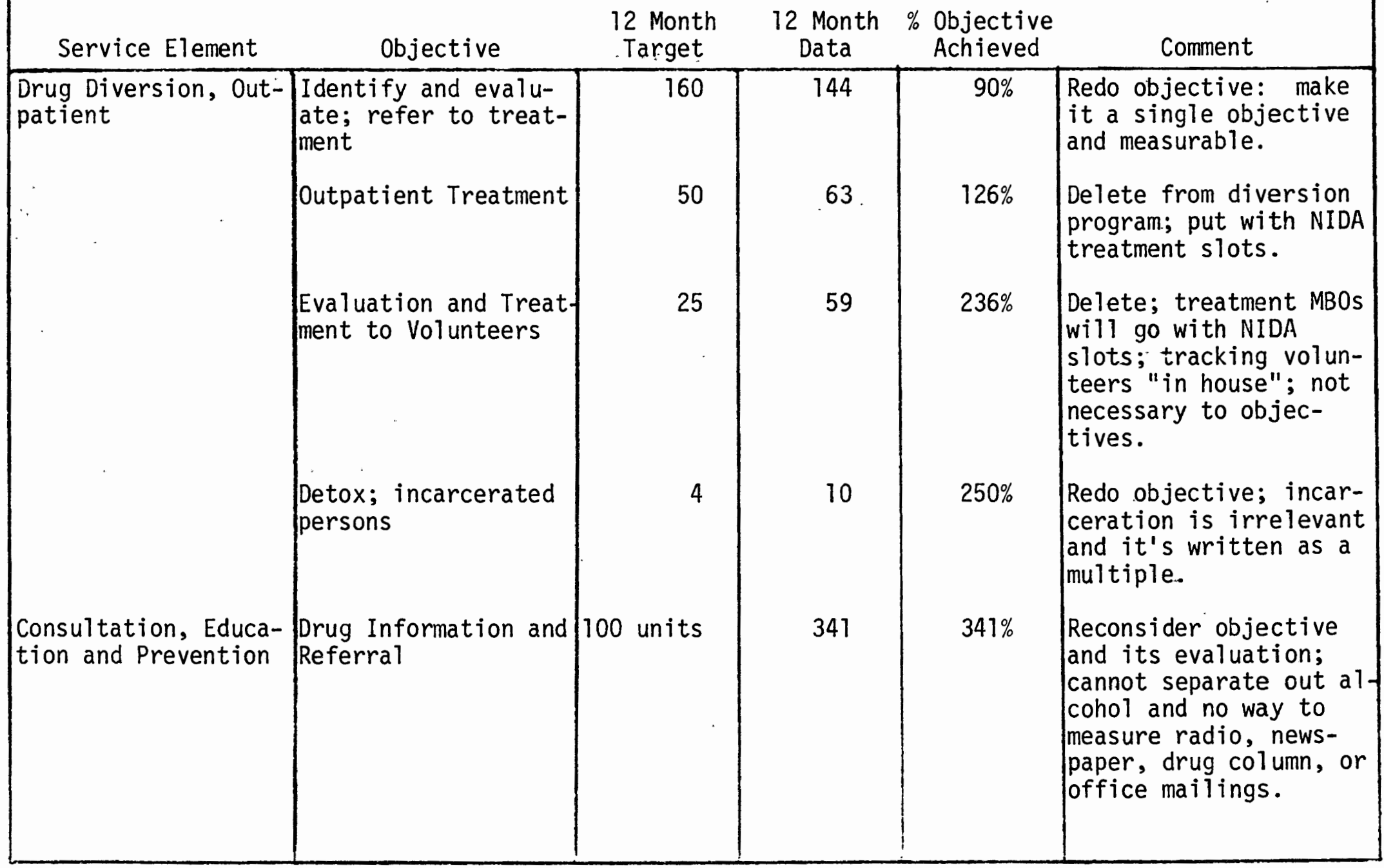




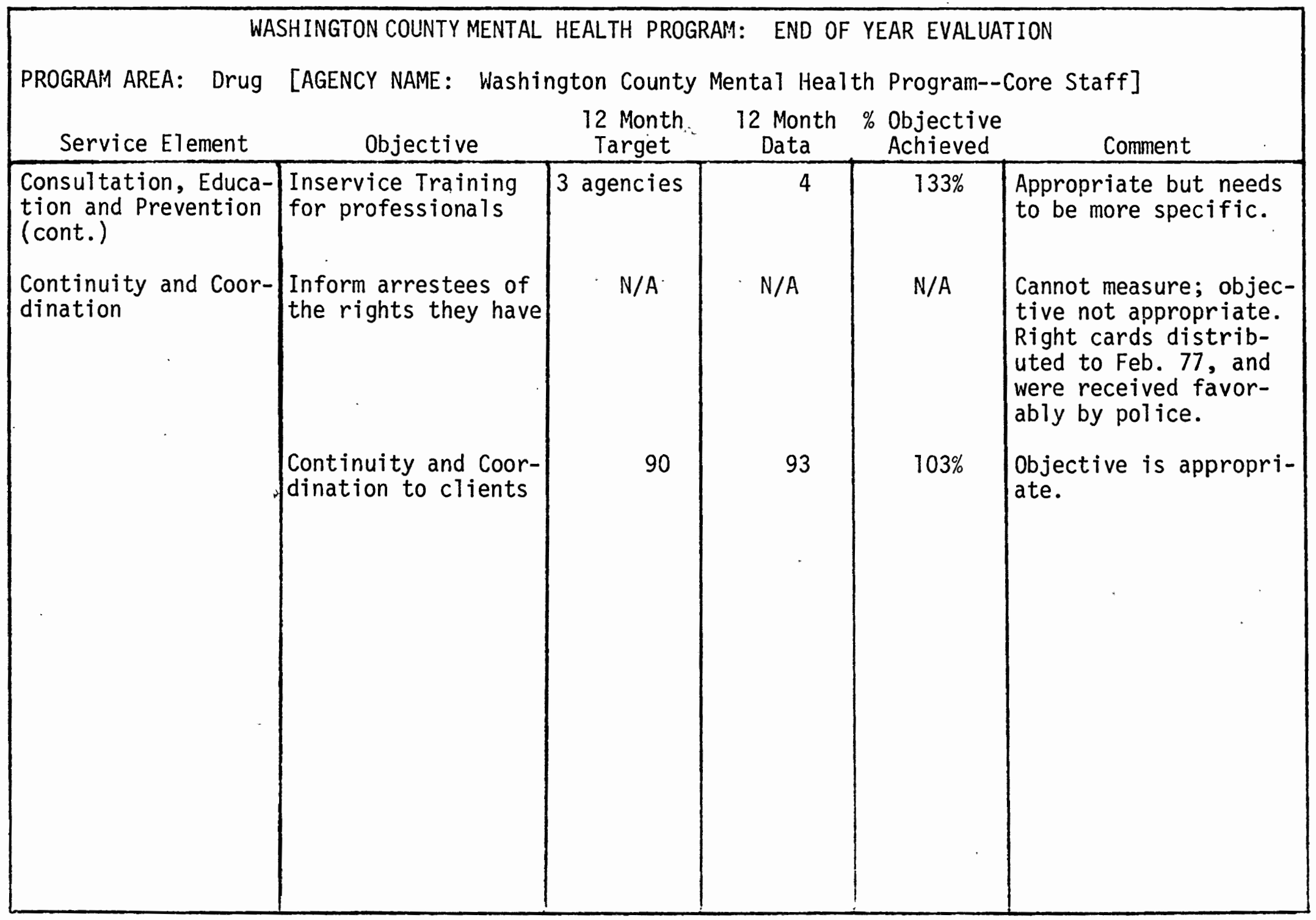

ज् 


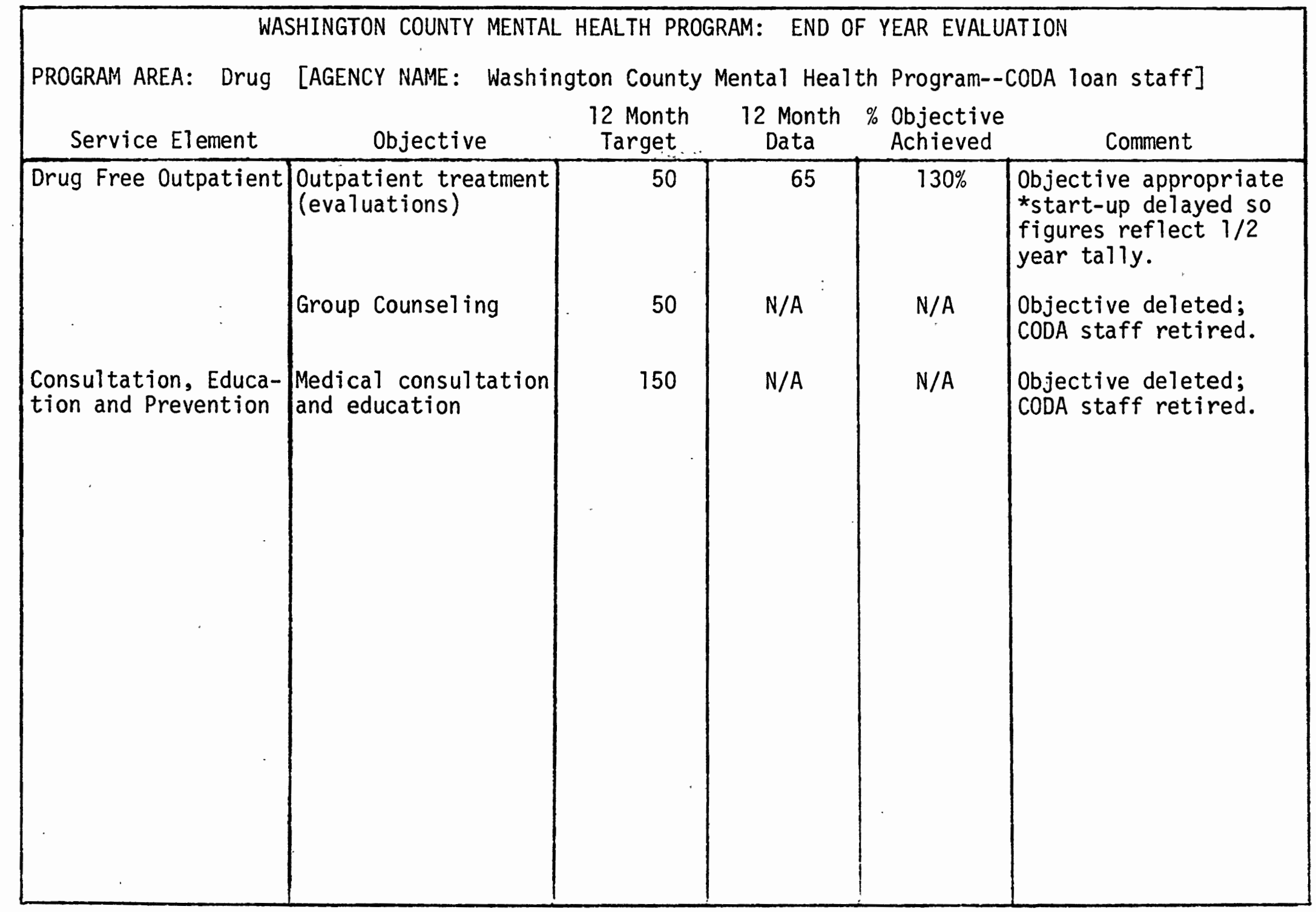




\begin{tabular}{|c|c|c|c|c|c|}
\hline & SHINGTON COUNTY MENTAL & HEALTH PROGI & END 0 & F YEAR EVALU & ATION \\
\hline PROGRAM AREA: Devel & opmental Disabilities & & & & \\
\hline Service Element & Objective & $\begin{array}{l}12 \text { Month } \\
\text { Target } \\
\end{array}$ & $\begin{array}{l}12 \text { Month } \\
\text { Data }\end{array}$ & $\begin{array}{c}\% \text { Objective } \\
\text { Achieved }\end{array}$ & Comment \\
\hline Beaverton Preschool & $\begin{array}{l}\text { To provide early in- } \\
\text { tervention for } 36 \\
\text { children }\end{array}$ & 36 & 38 & $105 \%$ & $\begin{array}{l}\text { Need funding for } 1 \text { ad- } \\
\text { ditional classroom, } \\
\text { but it is not avail- } \\
\text { able. }\end{array}$ \\
\hline Beaverton TMR & $\begin{array}{l}\text { To provide classroom } \\
\text { services for } 103 \\
\text { children }\end{array}$ & 103 & 93 & $90 \%$ & $\begin{array}{l}\text { Funding for FY } 78 \text { has } \\
\text { been reduced to pro- } \\
\text { vide services for } 84 \\
\text { children. Forest } \\
\text { Grove will provide ad- } \\
\text { ditional services for } \\
\text { TMR students. }\end{array}$ \\
\hline Forest Grove TMR & $\begin{array}{l}\text { To provide classroom } \\
\text { services for up to } \\
12 \text { children }\end{array}$ & 12 & 10 & $83 \%$ & $\begin{array}{l}\text { Within funding guide- } \\
\text { lines. }\end{array}$ \\
\hline Good Shepherd TMR & $\begin{array}{l}\text { To provide classroom } \\
\text { services for } 19 \\
\text { children }\end{array}$ & 19 & 16 & $84 \%$ & $\begin{array}{l}\text { Within funding guide- } \\
\text { lines. }\end{array}$ \\
\hline $\begin{array}{l}\text { Edwards Activity } \\
\text { Center }\end{array}$ & Serve 48 adults & 48 & 40 & $83 \%$ & $\begin{array}{l}\text { Objective has been re- } \\
\text { duced due to lack of } \\
\text { funds. }\end{array}$ \\
\hline & $\begin{array}{l}\text { Place } 10 \text { clients in } \\
\text { more appropriate } \\
\text { program }\end{array}$ & 10 & 4 & $40 \%$ & Objective too high. \\
\hline
\end{tabular}




\begin{tabular}{|c|c|c|c|c|c|}
\hline \multicolumn{6}{|c|}{$\begin{array}{l}\text { WASHINGTON COUNTY MENTAL HEALTH PROGRAM: } \\
\text { PROGRAM AREA: Developmental Disabilities }\end{array}$} \\
\hline $\begin{array}{l}\text { Edwards Activity } \\
\text { Center. } \\
\text { (cont.) }\end{array}$ & $\begin{array}{l}\text { Secure } 10-15 \text { job } \\
\text { training packages } \\
3-4 \text { parent meetings }\end{array}$ & $\begin{array}{l}15 \\
4\end{array}$ & 2 & $\begin{array}{r}233 \% \\
50 \%\end{array}$ & $\begin{array}{l}\text { Two meetings is more } \\
\text { realistic. }\end{array}$ \\
\hline Edwards Group Home & $\begin{array}{l}\text { To provide a resi- } \\
\text { dential program for } \\
8-12 \text { residents }\end{array}$ & $8-12$ & 10 & $100 \%$ & \\
\hline TVW Activity Center & $\begin{array}{l}\text { Provide program for } \\
24 \text { clients }\end{array}$ & 24 & 24 & $100 \%$ & \\
\hline $\begin{array}{l}\text { Child Development } \\
\text { Program }\end{array}$ & & 85 & 106 & $125 \%$ & $\begin{array}{l}77-78 \text { objectives have } \\
\text { been increased. }\end{array}$ \\
\hline $\begin{array}{l}\text { Services Coordina- } \\
\text { tion }\end{array}$ & $\begin{array}{l}\text { Yearly caseload of } \\
100 \text { clients } \\
\text { Consult monthly with } \\
15 \text { agencies } \\
\text { Fol low along for } \\
250 \text { clients }\end{array}$ & 100 & $\begin{array}{c}400 \\
\text { Unknown }\end{array}$ & $110 \%$ & $\begin{array}{l}\text { This objective will no } \\
\text { longer be used; } 77-78 \\
\text { objectives have been } \\
\text { modified. }\end{array}$ \\
\hline
\end{tabular}




\section{WASHINGTON COUNTY MENTAL HEALTH PROGRAM: END OF YEAR EVALUATION}

PROGRAM AREA: Mental and Emotional Disturbance

Service Element

1) Outpatient counseling services

2) Mental health homemaker services

3) Brief inpatient services

4) Day Treatment services

5) Education/ socialization services

\begin{tabular}{|c|c|c|c|c|}
\hline Objective & $\begin{array}{l}12 \text { Month } \\
\text { Target } . .\end{array}$ & $\begin{array}{l}12 \text { Month } \\
\text { Data }\end{array}$ & $\begin{array}{c}\% \text { Objective } \\
\text { Achieved }\end{array}$ & Comment \\
\hline $\begin{array}{l}\text { To provide individ- } \\
\text { ual, marital and } \\
\text { family counseling to } \\
1,403 \text { Washington } \\
\text { County residents }\end{array}$ & 1,403 & 1,582 & $113 \%$ & $\begin{array}{l}\text { Objectives should be } \\
\text { increased; a trend to- } \\
\text { ward increased utili- } \\
\text { zation of these ser- } \\
\text { vices is noted. }\end{array}$ \\
\hline $\begin{array}{l}\text { To maintain } 20-50 \\
\text { pre- or post- } \\
\text { hospital clients in } \\
\text { their own homes }\end{array}$ & 20 & 14 & $90 \%$ & $\begin{array}{l}\text { Objectives were low- } \\
\text { ered due to lack of } \\
\text { staff. }\end{array}$ \\
\hline $\begin{array}{l}\text { To provide short- } \\
\text { term inpatient ser- } \\
\text { vices to } 15 \text { Washing- } \\
\text { tion County resi- } \\
\text { dents }\end{array}$ & 15 & 19 & $127 \%$ & $\begin{array}{l}\text { Went over budget } 4,77 \text {; } \\
\text { increased funding } \\
\text { should now meet need. }\end{array}$ \\
\hline $\begin{array}{l}\text { To provide day } \\
\text { treatment services } \\
\text { to } 60 \text { Washington } \\
\text { County residents }\end{array}$ & 60 & 52 & $86 \%$ & $\begin{array}{l}\text { Program is expanding } \\
\text { rapidly; planning and } \\
\text { individual appoint- } \\
\text { ments reduced direct } \\
\text { treatment services. }\end{array}$ \\
\hline $\begin{array}{l}\text { To provide educa- } \\
\text { tional services, in } \\
\text { the spirit of pre- } \\
\text { vention, to } 630 \\
\text { Washington County } \\
\text { residents }\end{array}$ & 630 & 264 & $42 \%$ & $\begin{array}{l}\text { Data collection was } \\
\text { inconsistent; plans } \\
\text { for } 1977-1978 \text { include } \\
\text { strengthening these } \\
\text { services. }\end{array}$ \\
\hline
\end{tabular}


WASHINGTON COUNTY MENTAL HEALTH PROGRAM: END OF YEAR EVALUATION

PROGRAM AREA: Mental and Emotional Disturbance

Service Element

$\begin{array}{cccc}12 \text { Month } & \begin{array}{c}12 \text { Month } \% \text { Objective } \\ \text { Data }\end{array} & \begin{array}{c}12 \text { Achieved } \\ \text { Target }:\end{array} \text { Comment }\end{array}$

6) Consultation ser- To provide 704 hrs. vices

of consultation services to Washington County agencies

7) Commitment/ diversion

To provide services as needed: Washington County involuntary clients. Service categories as follows:

1) Info. \& Referral

2) Investigations

704

850

Achieved

Objectives need to be further specified and increased.

Objectives for this area must be quantified. There have been fewer inappropriate petitions filed this year, compared to last year, due to an increase in staff expertise and a greater number of diverted $\mathrm{cli}$ -

3) Hearings

4) Commitment proceedings

8) Continuity and Coordination/ Alternatives to To provide alternative services to Washington County

AS commitment program residents ents due to the rapidly expanding alternative program.

This has been an active, growing program area. Objectives will be outlined for 19771978. 


\begin{tabular}{|c|c|c|c|c|c|}
\hline $\begin{array}{l}\text { PROGRAM AREA: Ment } \\
\text { Service Element }\end{array}$ & \multicolumn{5}{|c|}{$\begin{array}{l}\text { ASHINGTON COUNTY MENTAL HEALTH PROGRAM: END OF YEAR EVALUATION } \\
\text { al and Emotional Disturbance [AGENCY NAME: Cedar Hills Hospital] }\end{array}$} \\
\hline Brief Inpatient & $\begin{array}{l}\text { Short-term inpatient } \\
\text { services to } 15 \text { cli- } \\
\text { ents } \\
\text { a) Number of clients } \\
\text { served } \\
\text { b) Commitment pro- } \\
\text { cedures } \\
\text { c) Length of hospi- } \\
\text { talization } \\
\\
\text { 215 hr. M.D. ser- } \\
\text { vices to inpatient } \\
\text { clients } \\
\text { a) Number of clients } \\
\text { served } \\
\text { b) Total Client } \\
\text { hours }\end{array}$ & $\begin{array}{l}\text { None speci- } \\
\text { fied } \\
3-7 \text { day } \\
\text { average }\end{array}$ & $\begin{array}{l}19 \\
21 \\
21 \\
106.5 \text { total } \\
\text { days } \\
5.6 \text { day } \\
\text { average }\end{array}$ & $127 \%$ & $\begin{array}{l}\text { Went over budget end } \\
\text { of April. Increased } \\
\text { funding } 77-78 \text { should } \\
\text { more realistically } \\
\text { meet need. } \\
\text { Four of } 19 \text { clients } \\
\text { were in hospital up to } \\
12 \text { day limit ( } 1 \text { pa- } \\
\text { tient had } 15 \text { days). } \\
12 \text { day limitation ap- } \\
\text { pears to be generally } \\
\text { adequate. } \\
\\
\text { objective too high. } \\
\text { Should allocate approx- } \\
1.0 \text { hr/day/client, } \\
\text { i.e., for the } 19 \text { cli- } \\
\text { ents served this year }\end{array}$ \\
\hline
\end{tabular}




\begin{tabular}{|c|c|c|c|c|c|}
\hline \multicolumn{6}{|c|}{$\begin{array}{l}\text { WASHINGTON COUNTY MENTAL HEALTH PROGRAM: END OF YEAR EVALUATION } \\
\text { PROGRAM AREA: Mental and Emotional Disturbance [AGENCY NAME: Cedar Hills Hospital] }\end{array}$} \\
\hline $\begin{array}{l}\text { Brief Inpatient } \\
\text { (cont.) }\end{array}$ & $\begin{array}{l}\text { b) Total Client } \\
\text { hours } \\
\text { (cont.) } \\
\text { c) Type client serve } \\
\text { served } \\
\text { d) Post-hospital } \\
\text { courses } \\
\text { e) Range of services } \\
\text { 100 hr. M.D. consul- } \\
\text { tation } \\
\text { a) Total hours in- } \\
\text { volvement }\end{array}$ & 100 & $\begin{array}{l}18 \text { volunta- } \\
\text { ry; } 12 \text { com- } \\
\text { mitted }\end{array}$ & $122+\%$ & $\begin{array}{l}\text { with } 5.6 \text { day average } \\
\text { stay }=106 \text { hours } 11 . D \text {. } \\
\text { services. } \\
\\
\text { Consultation heavily } \\
\text { utilized by core staff } \\
\text { (and in some cases } \\
\text { other agencies) An } \\
\text { additional } 75+\text { hours } \\
\text { of consultation was } \\
\text { possible through unex- } \\
\text { pended ICP dollars; } \\
\text { making a total of } 200 \\
\text { hours consultation, } \\
\text { i.e. consultation ob- } \\
\text { jective should be in- } \\
\text { creased. }\end{array}$ \\
\hline
\end{tabular}


WASHINGTON COUNTY MENTAL HEALTH PROGRAM: END OF YEAR EVALUATION

PROGRAM AREA: Mental and Emotional Disturbance [AGENCY NAME: Lutheran Family Service]

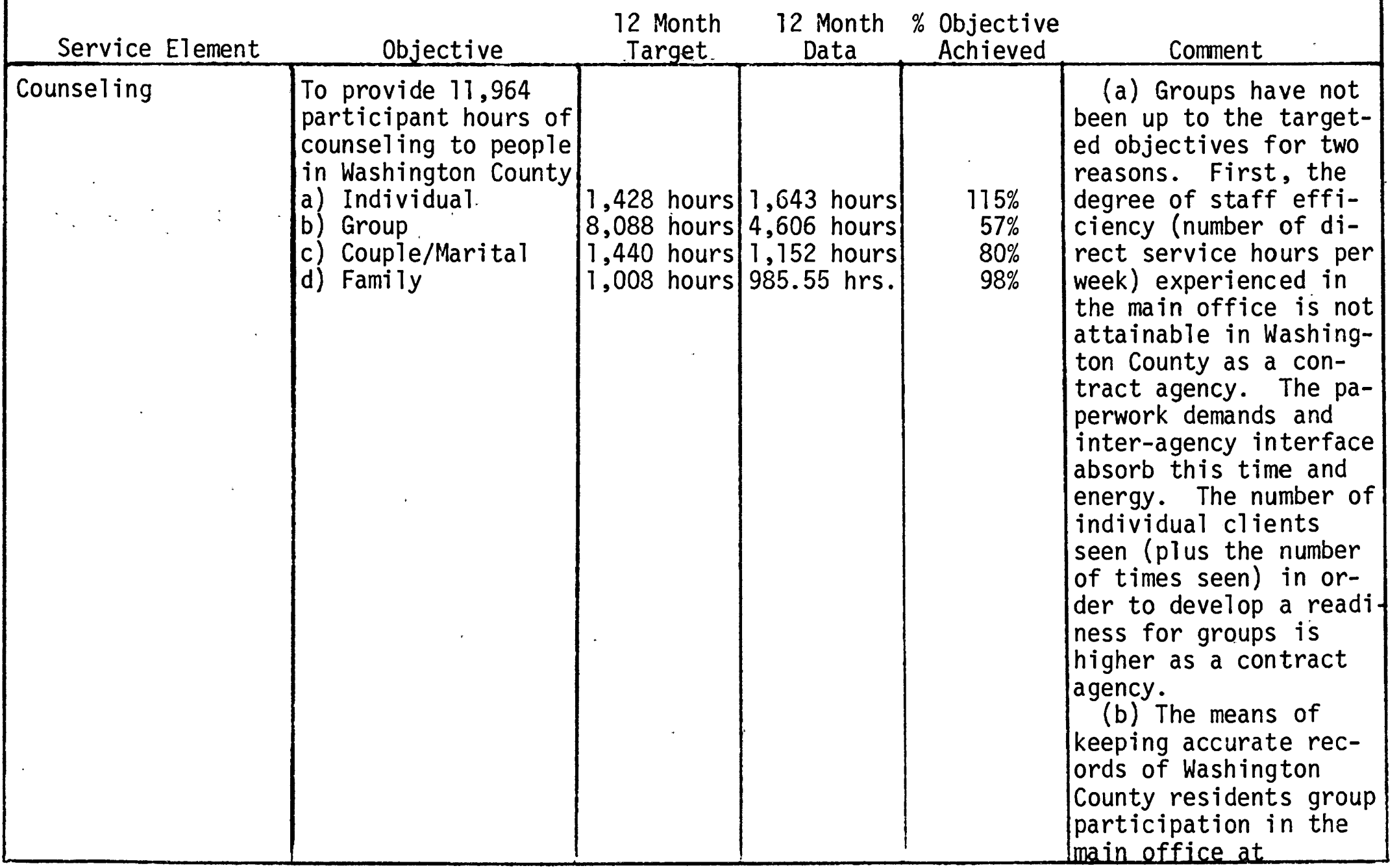

$\omega$ 


\begin{tabular}{|c|c|c|c|c|c|}
\hline \multicolumn{6}{|c|}{$\begin{array}{l}\text { WASHINGTON COUNTY MENTAL HEALTH PROGRAM: END OF YEAR EVALUATION } \\
\text { PROGRAM AREA: Mental and Emotional Disturbance [AGENCY NAME: Lutheran Family Service] } \\
\begin{array}{ccccc}\text { Objective } & 12 \text { Month } 12 \text { Month } \% \text { objective } \\
\text { Service Element } & \text { Target } & \text { Data } & \text { Achieved } & \text { Comment }\end{array}\end{array}$} \\
\hline $\begin{array}{l}\text { Counseling } \\
\text { (cont.) }\end{array}$ & $\begin{array}{l}\text { To provide } 11,964 \\
\text { participant hours of } \\
\text { counseling to people } \\
\text { in Washington County } \\
\text { (cont.) }\end{array}$ & & & & $\begin{array}{l}\text { Multnomah Courity has } \\
\text { only been perfected } \\
\text { in the last five. } \\
\text { months. }\end{array}$ \\
\hline Life Education & $\begin{array}{l}\text { To provide classes } \\
\text { for parents, teens, } \\
\text { and people wanting } \\
\text { to change some of } \\
\text { their behaviors } \\
\text { a) TA for Parents } \\
\text { b) PET } \\
\text { c) TA for Teens } \\
\text { d) Tools for Change } \\
\text { e) Change Now } \\
\text { f) Divorce Seminars }\end{array}$ & $\begin{array}{l}1 \text { class } \\
2 \text { classes } \\
4 \text { classes } \\
6 \text { classes } \\
11 \text { classes } \\
3 \text { classes }\end{array}$ & $\begin{array}{l}0 \\
3 \\
2 \\
5 \\
1 \\
1\end{array}$ & $\begin{array}{c}100 \% \\
150 \% \\
50 \% \\
80 \% \\
-- \\
33 \%\end{array}$ & $\begin{array}{l}\text { Objectives developed } \\
\text { before staff changes } \\
\text { required a reduced em- } \\
\text { phasis on TA for } \\
\text { Teens. In addition, } \\
\text { scheduled classes did } \\
\text { not fill; probably } \\
\text { need more comprehen- } \\
\text { sive advertising. The } \\
\text { Tools for Change and } \\
\text { Divorce Seminars } \\
\text { classes were scheduled } \\
\text { but not filled; again, } \\
\text { probable need for } \\
\text { broader advertising } \\
\text { coverage. Change Now } \\
\text { held in main office. } \\
\text { No statistics were } \\
\text { recorded. }\end{array}$ \\
\hline
\end{tabular}




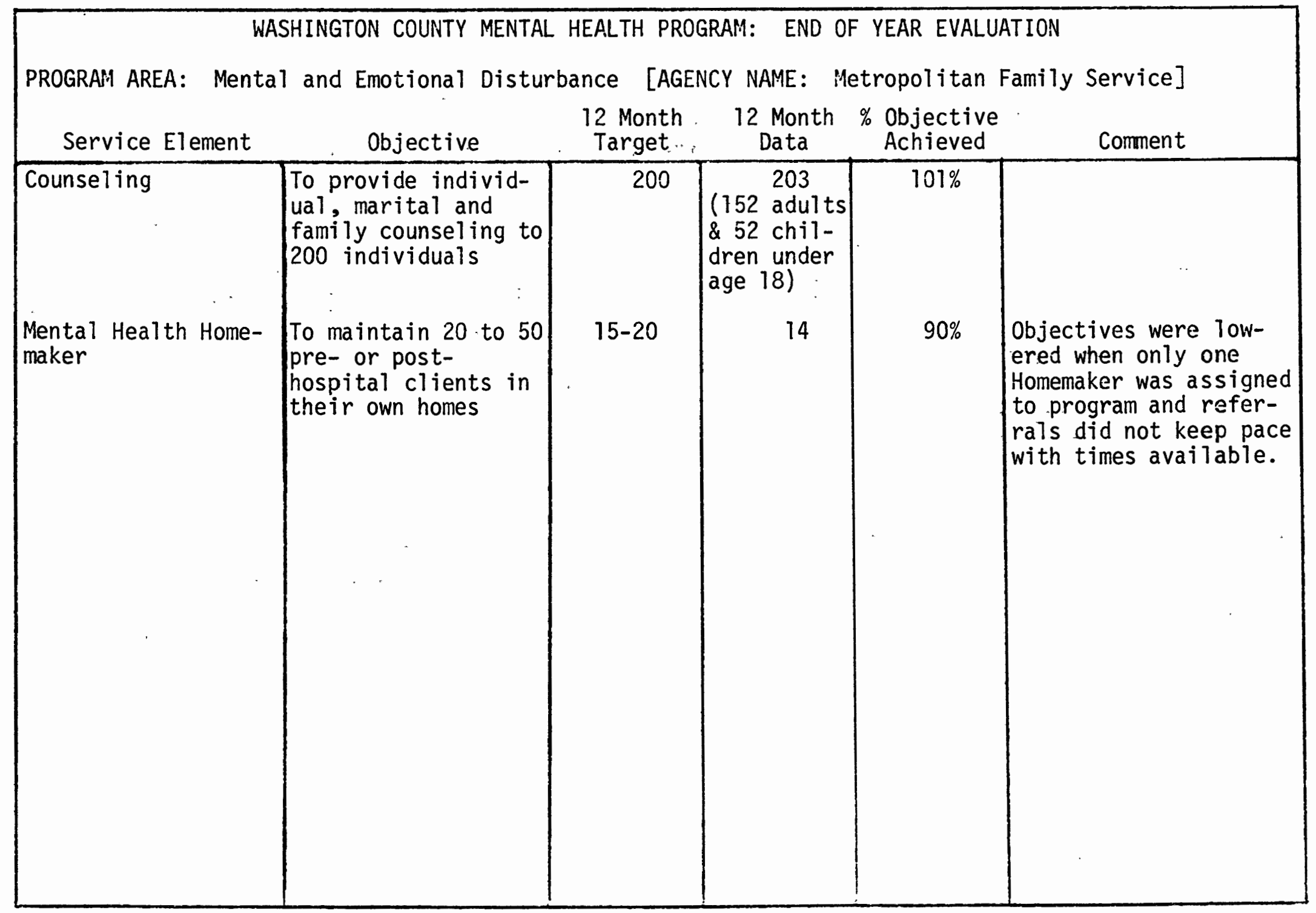




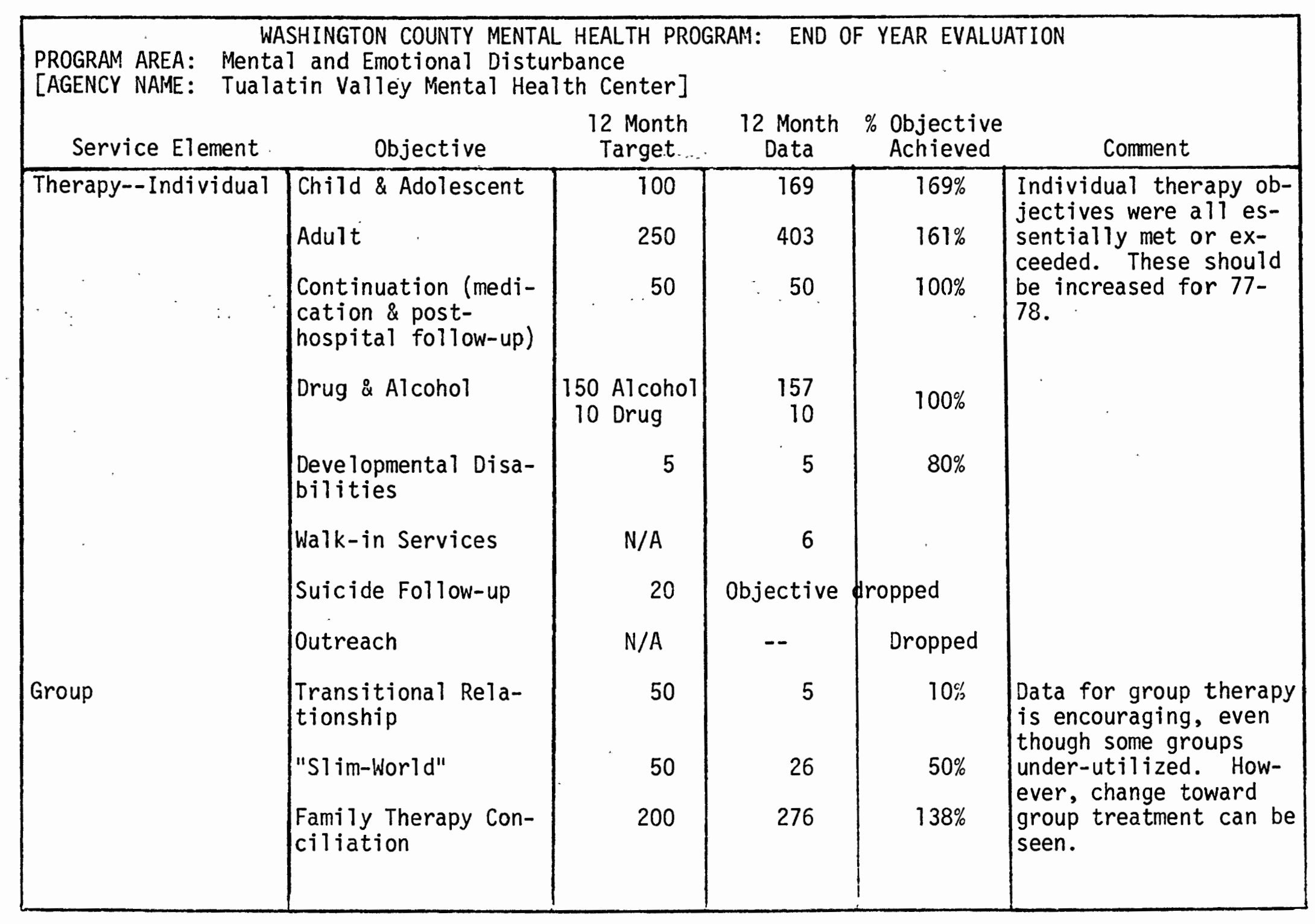




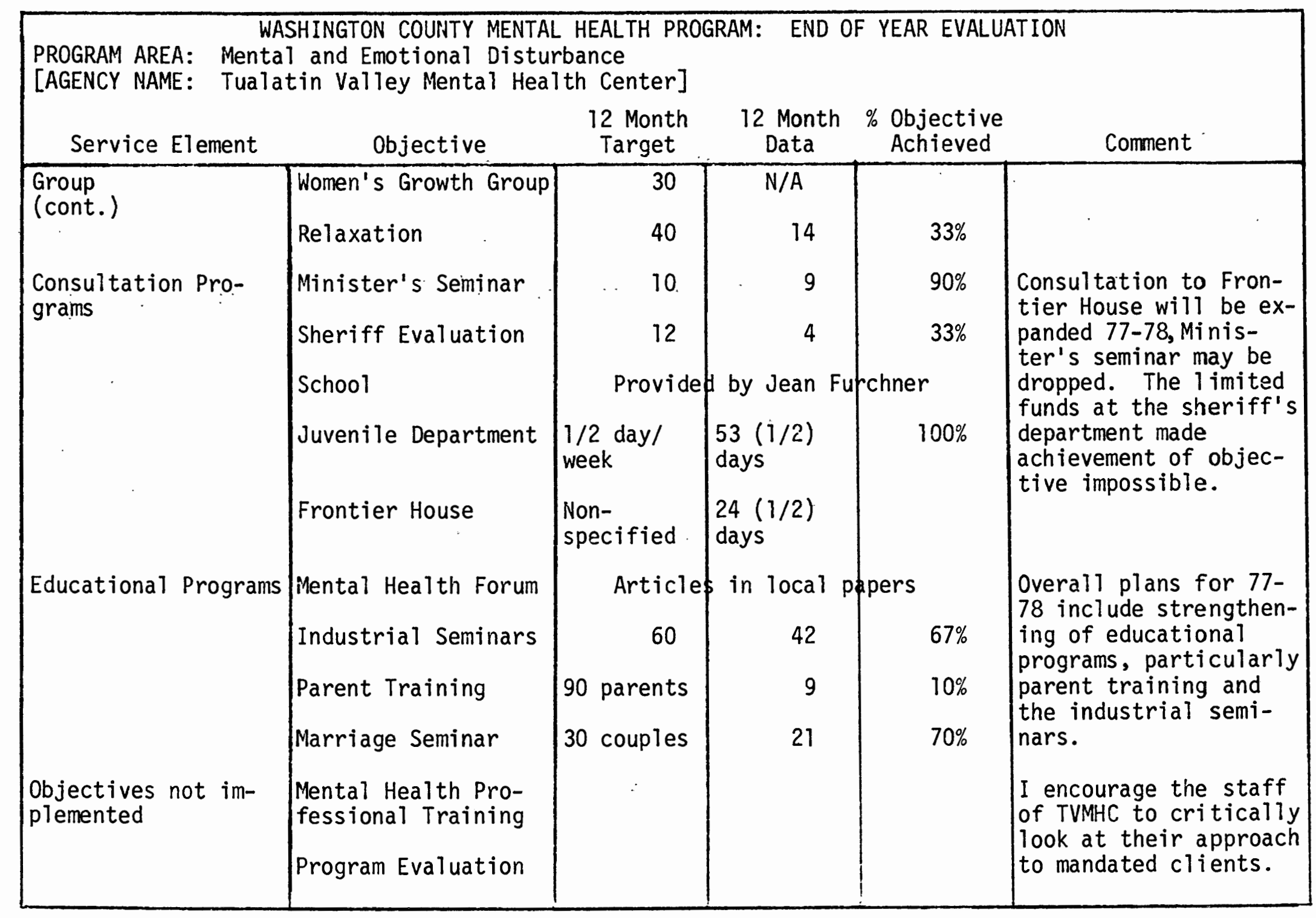




$$
\text { Fime }
$$




\begin{tabular}{|c|c|c|c|c|c|}
\hline \multicolumn{6}{|c|}{ 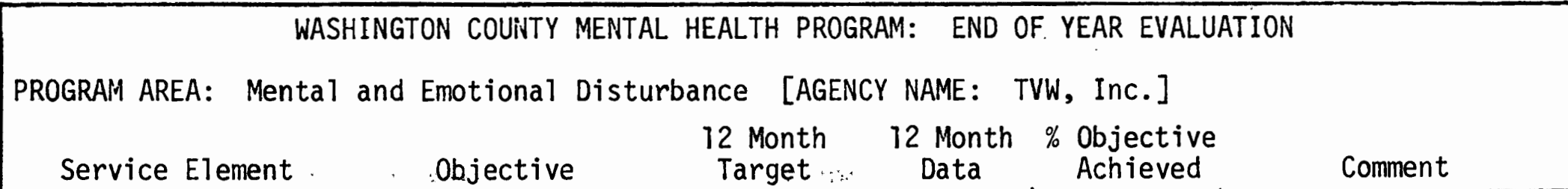 } \\
\hline Day Treatment & \begin{tabular}{|l|} 
Day treatment ser- \\
vices to 60 clients \\
\\
Increase length of \\
time in community of \\
$80 \%$ clients served \\
Increase life and \\
socialization skills \\
for 40 clients \\
More clients to paid \\
employment opportunif \\
ties
\end{tabular} & $\begin{array}{l}6 \\
60\end{array}$ & 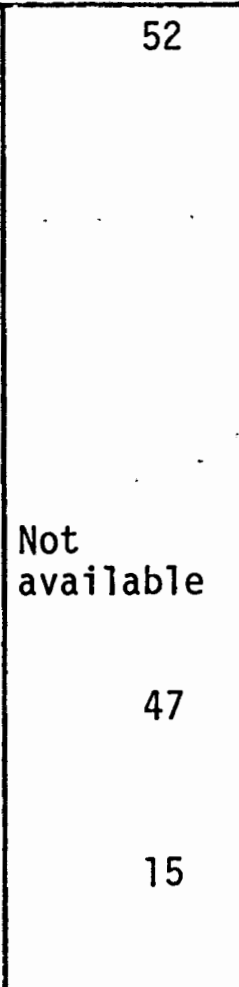 & $\begin{array}{l}117 \% \\
250 \%\end{array}$ & $\begin{array}{l}\text { Program grown tremen- } \\
\text { dously in last six } \\
\text { months. Now serving } \\
5.8 \text { FTE (7/77). Fig- } \\
\text { ure would likeiy have } \\
\text { been higher; however, } \\
\text { planning \& individual } \\
\text { appointments (for } \\
\text { follow-up) reduced di- } \\
\text { rect day treatment } \\
\text { services. } \\
\\
\text { Difficulties with CL-1 } \\
\text { outcome evaluation un- } \\
\text { realistic. }\end{array}$ \\
\hline
\end{tabular}




\begin{tabular}{|c|c|c|c|c|c|}
\hline \multicolumn{6}{|c|}{ 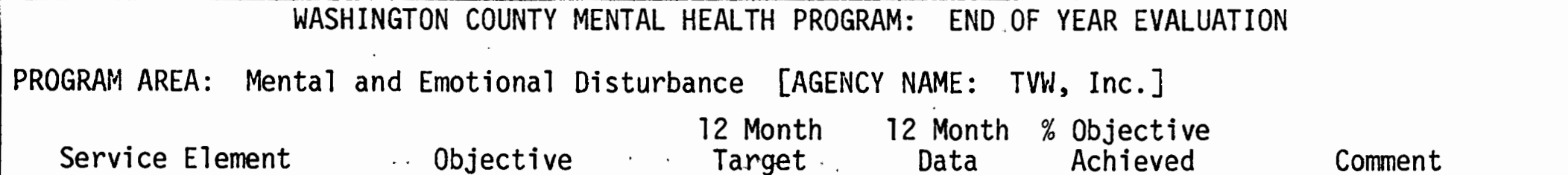 } \\
\hline $\begin{array}{l}\text { Socialization Pro- } \\
\text { gram }\end{array}$ & $\begin{array}{l}\text { Socialization pro- } \\
\text { gramming to MED and } \\
\text { MR/DD clients } \\
\text { Home visits } \\
\text { Consultation for en- } \\
\text { vironmental improve- } \\
\text { ment }\end{array}$ & $\begin{array}{l}900 \text { units } \\
\text { of service } \\
100 \text { undup- } \\
\text { licated } \\
\text { clients } \\
\text { counsel } 80 \\
80 \% \text { CL-1 } \\
\text { improve } \\
150 \text { units } \\
20\end{array}$ & 36 & $72 \%$ & $\begin{array}{l}\text { Therapy and socializa- } \\
\text { tion units of service } \\
\text { should be in a com- } \\
\text { bined objective. } \\
\text { Unrealistic goal; too } \\
\text { high. } \\
50 \text { clients would be } \\
\text { more realistic. } \\
\text { CL-l output through } \\
\text { MHIS does not yield } \\
\text { this data. } \\
\text { This objective dropped } \\
\text { from } 77-78 \text { program. } \\
\text { This objective dropped } \\
\text { from } 77-78 \text { program. }\end{array}$ \\
\hline
\end{tabular}


WASHINGTON COUNTY MENTAL HEALTH PROGRAM: END OF YEAR EVALUATION

PROGRAM AREA: Mental and Emotional Disturbance [AGENCY NAME: TVW, Inc.]

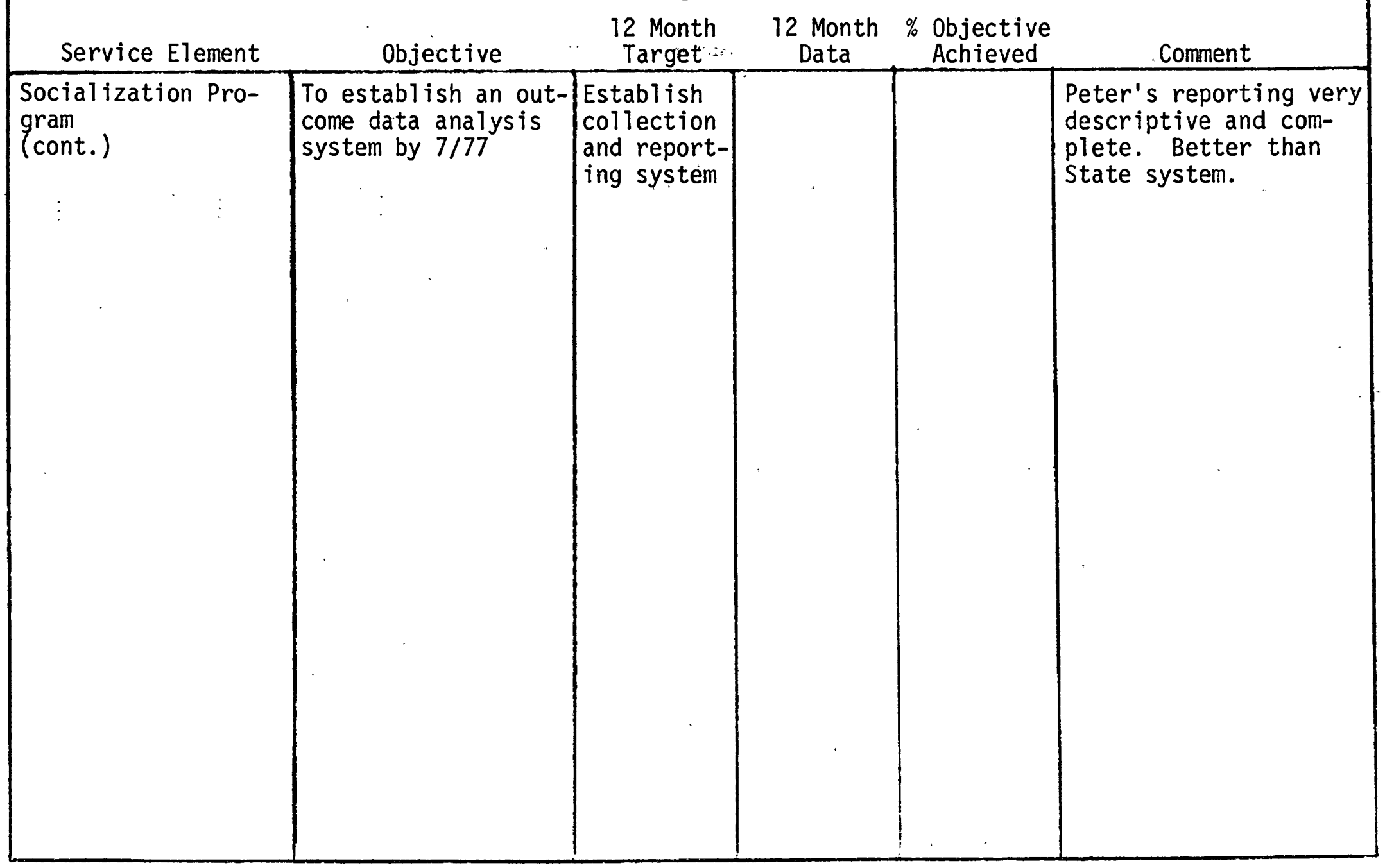

오 
CHAPTER III

\section{CONCLUSIONS AND RECOMMENDATIONS}

There are some benefits to this type of evaluation; the results indicate current service utilization rates and emphasize accountability at the agency level. As mental health systems move toward "purchase of service" agreements with local agencies, data of this sort will be invaluable when it comes to identifying which agencies to contract with for which services. In addition, the results are useful tools in lobbying efforts with the state legislature and other funding sources. It is easy to glean, from the Year End Report, what types of target populations are being served (and what types are not receiving adequate services) as well as which service elements are over/underutilized.

There are obvious limitations associated with this type of evaluation design. The first problem stems from the fact that it is not ongoing, in the sense that there is no continual recycling of information, but rather a once-a-year report. Although the $\mathrm{CL}-1$ reports are actualiy written two times a year, this also is not frequent enough, in the opinion of this author, to be termed ongoing evaluation. Although the reports are useful to the program director, the actual service providers receive little if any information concerning their daily activities. The information that they do receive is not imparted with a program remodeling flavor but rather an after-the-fact spirit. One would expect a good evaluation system to provide service delivery agents with regular 
information concerning the treatment (and effects of that treatment) that they are providing. Further, the information would be imparted at closer intervals (weekly, monthly, quarterly) in order that it could be useful in terms of decision making at many levels (therapist, agency, program).

At this point the only form of ongoing evaluation, taking place with any degree of regularity and designed to be responsive to the professional needs of the service delivery staff, is peer review and utilization review. Both of these evaluation activities are sporadic, nonsystematic, and are often perceived as punitive in nature rather than educational (as is their intent).

\section{MHIS}

Another problem associated with the $\mathrm{CL}-1$ and annual reports relates to the validity and reliability of the data collection system. The MHIS, a statewide service available to all public local mental health programs, has serious drawbacks. The CL-1 form itself, which is the data collection instrument as far as the state MHIS is concerned, is outdated, dysfunctional, unreliable and, at best, incomplete. A copy of this form has been included in this research practicum for purposes of illustration. It will be found in the Appendix.

The form is often not filled out accurately by the service deliverers. Many of these clinicians approach the $\mathrm{CL}-1$ form as a worthless chore that only detracts from the amount of time they could put to use in actual service delivery, a folly of the administration. Most of the data that are collected do not correspond to issues that the service 
providers are concerned with, such as the efficacy of treatment models, the relative success of their personal approaches, etc. Many providers fill out the form in a standard way for each of their clients, noting no differences between clients with regard to diagnosis, the progression of treatment and outcome; many do not ever complete the $\mathrm{CL}-1$ form for their clients. Thus, at the very base level, the foundation of the information system, the information going into the MHIS is incomplete and frequently inaccurate.

There are also structural problems with the form. For example, it seems unrealistic to break client income down by intervals of $\$ 1,000$; most clients find it difficult if not impossible to estimate income with that degree of accuracy. Beyond that, what real, significant difference is there between a client earning $\$ 6,000$ a year and a similar client earning $\$ 7,000$ a year in 1977? A revision of such structural problems associated with the $\mathrm{CL}-1$ form is long overdue.

Yet another source of frustration associated with the state MHIS is the type of information collected. Most service providers and administrators alike plead to have "number of dependents," "source of income," and "marital status" indicators replaced with indicators that will reflect the outcome of treatment. As the $\mathrm{CL}-1$ form stands, much may be extracted regarding the quantity of treatment and little may be said concerning the quality of treatment. Thus the MHIS dictates process evaluation not outcome evaluation. 


\section{ALTERNATIVES TO MHIS}

During the past year, the Washington County Mental Health Program has sought alternatives to the state MHIS. They have seriously considered depending less upon the state MHIS and creating their own information system. Money was budgeted for a private consulting firm to examine the program, including evaluation needs, and to draft a model for a new information system. This model is now in the hands of Washington County mental health administrators. The major considerations associated with the move from the state MHIS to the new model can be boiled down to: "It is more cost effective (cheaper) to stay with the state system" versus "It is more valuable, in terms of quality of treatment, to go with the new model." This issue is currently under close examination; however, no decision has been made. Other counties in Oregon have made the decision to develop and implement their own information systems and have capitalized by gaining a more program responsive evaluation model.

\section{DECISION-MAKING PROCESS}

Returning to the $\mathrm{CL}-1$ reports and the annual reports and looking at the decision-making structure in Washington County Mental Health, it must be reemphasized that, while other options are being considered, these reports are presently the major evaluation actitivites conducted within the agency. The results concluded in these studies, therefore, as they relate to the $\mathrm{MBOS}$, will determine future funding for the program from both state and local funding sources. These results will also be the basis for future modifications within the program as reflected in 
the further development and refinement of management objectives. The administrative staff will seek to modify these objectives in order to more closely approximate a 100 percent meeting of treatment objectives and actual treatment. For example, if one treatment objective reads, "We will provide brief inpatient treatment for twenty Washington County residents," and only ten residents actually received this service, representing only a 50 percent achievement of the original objective, then the objective will be revised the following year. In some cases the objective will remain the way. it is and the treatment approach will be revised. The goal is always a 100 percent match between projected goals and actual services delivered. As the evaluation system grows and is refined, it will become easier to control discrepancies.

Decision making comes mostly from the top down with some input, on treatment-related issues, from the service providers. The leverage point, as far as decision making is concerned, is based on funding, money. The people to be satisfied, as far as evaluation results are concerned, are the funding sources, the people with the money.

As most of the treatment providers in the county system are private and private-nonprofit agencies, subcontracted with Washington County Mental Health to provide specific services, their decision-making structures are discrete. Insofar as they are under contract with the county system, they are subject to accountability regarding their adherence to mutually agreed-upon treatment and management objectives. A subcontract agency that repeatedly fails to meet outlined objectives may. not be as generously funded the following year. 
RECOMMENDATIONS FOR CHANGE

While still hooked into the state MHIS, it is possible to implement some changes in evaluation procedures. It is recommended that the following changes be investigated: (1) a move toward more frequent cycling of program evaluation information (weekly, monthly, quarterly), (2) an attempt to engage more service providers in evaluation activities and the decision-making procedures, (3) a move toward more inhouse evaluation activities (should the three needs assessments have been conducted by agency staff members, benefits such as familiarity with client populations would not have been sacrificed), (4) an attempt to present peer review and utilization review in a less threatening manner that will be more valuable to the clinicians, and (5) a consistent and concerted effort to link evaluation activities to specific decisions to avoid unnecessary wasted energy and paperwork.

As a more long-term goal, implementation of a management information system that is more responsive to the local programs is recommended. This new information system may permit the following evaluation changes to occur: (1) a development and recording of more treatmentoriented criteria (as opposed to demographic data only), (2) a development and recording of outcome data reflecting quality of treatment (as opposed to quantity of treatment only), and (3) an information recycling system that provides information to professionals at all treatment levels (as opposed to administration only). 
APPENDIX 


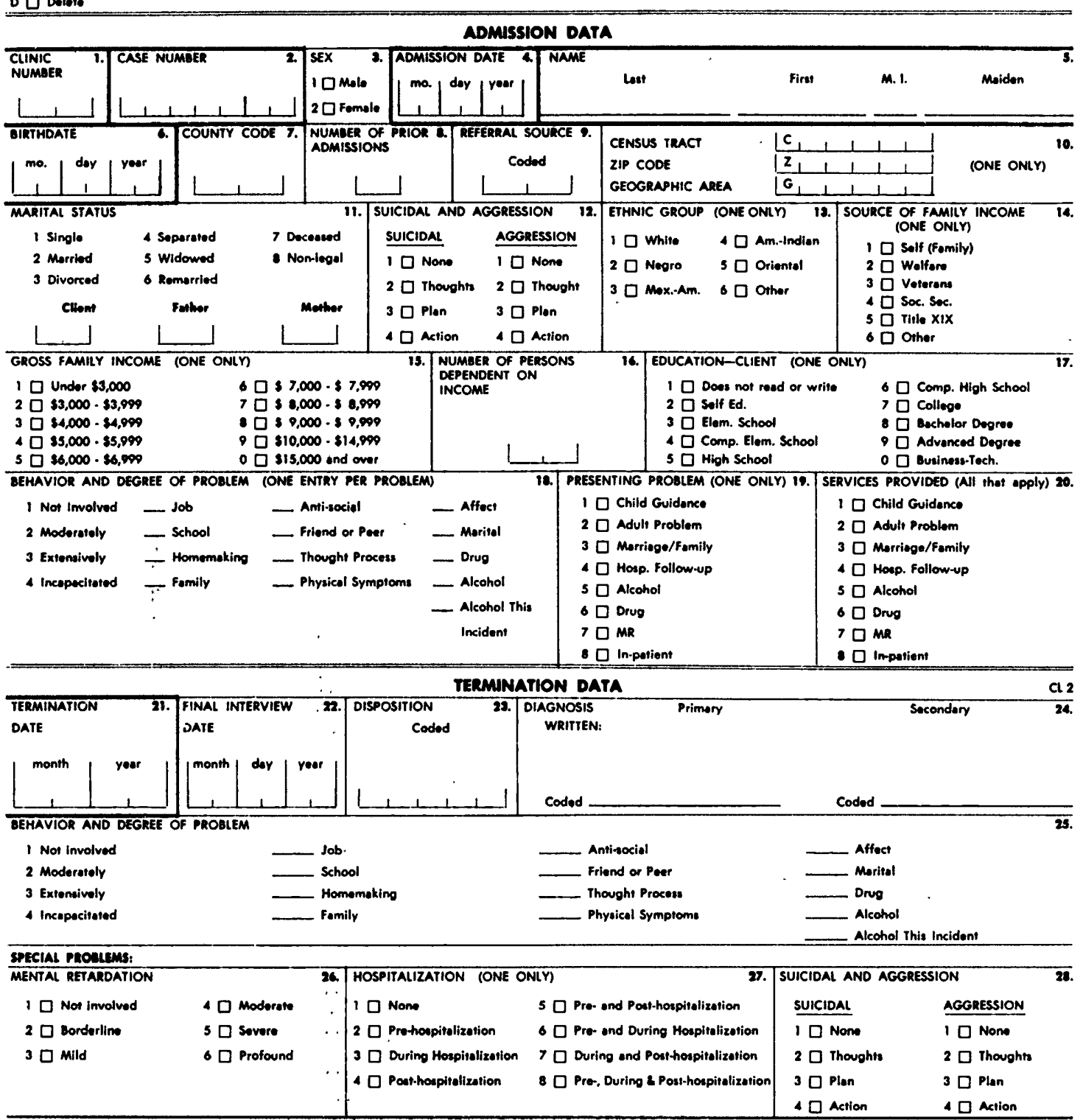

COMMENTS: 